\title{
The Bogoliubov-de Gennes system, the AKNS hierarchy, and nonlinear quantum mechanical supersymmetry
}

\author{
Francisco Correa ${ }^{\dagger}$, Gerald V. Dunne ${ }^{\ddagger}$ and Mikhail S. Plyushchay ${ }^{\dagger}$ \\ ${ }^{\dagger}$ Departamento de Fúsica, Universidad de Santiago de Chile, Casilla 307, Santiago 2, Chile \\ ${ }^{\ddagger}$ Physics Department, University of Connecticut, Storrs CT 06269, USA
}

\begin{abstract}
We show that the Ginzburg-Landau expansion of the grand potential for the Bogoliubov-de Gennes Hamiltonian is determined by the integrable nonlinear equations of the AKNS hierarchy, and that this provides the natural mathematical framework for a hidden nonlinear quantum mechanical supersymmetry underlying the dynamics.
\end{abstract}

\section{INTRODUCTION: THE BOGOLIUBOV-DE GENNES HAMILTONIAN}

The Bogoliubov-de Gennes (BdG) Hamiltonian [1] is

$$
H=\left(\begin{array}{cc}
-i \frac{d}{d x} & \Delta(x) \\
\Delta^{*}(x) & i \frac{d}{d x}
\end{array}\right),
$$

where the (in general complex) function $\Delta(x)$ plays the role of an order parameter, a condensate, or a gap function, depending on the physical context. The BdG Hamiltonian is relevant for the physics of: (i) crystalline condensates 2, 3, 4] in the chiral Gross-Neveu and Nambu-Jona Lasinio models [5, 6, 7, 8, 9]; (ii) fractional fermion number [10, 11]; (iii) the Peierls effect [12, 13]; (iv) polaron crystals in conducting polymers [14, 15, 16, 17]; (v) the pair potential in inhomogeneous quasi-1D superconductors [18, 19, 20, 21]; (vi) the order parameter for superconductors in a ferromagnetic field [22]; (vii) incommensurate charge-density waves [23, 24]; (viii) phase structure of ultracold atomic gases 25]. In this paper we discuss the rich mathematical physics properties of the BdG Hamiltonian and in particular its close relation to nonlinear quantum mechanical supersymmetry [26, 27].

An important object for the analysis of physical models based on the BdG Hamiltonian is the Gorkov resolvent [28, 29, 30, 31]:

$$
R(x ; E) \equiv\left\langle x\left|\frac{1}{H-E}\right| x\right\rangle
$$

The resolvent (1.2) is clearly a $2 \times 2$ matrix. It encodes the spectral properties of the BdG Hamiltonian. For example, the spectral function (density of states) characterizing the single-particle spectrum of fermions in the presence of the condensate $\Delta(x)$ is

$$
\rho(E)=\frac{1}{\pi} \operatorname{Im}_{\operatorname{Tr}}[R(x ; E+i \epsilon)]
$$

where the trace is a Dirac trace as well as a spatial trace. The local density of states is defined without the spatial trace:

$$
\rho(x ; E)=\frac{1}{\pi} \operatorname{Im}_{\operatorname{Tr}}[R(x ; E+i \epsilon)] .
$$

The thermodynamic grand potential density is defined as

$$
\psi(x)=-\frac{1}{\beta} \int_{-\infty}^{\infty} d E \rho(x ; E) \ln \left(1+e^{-\beta(E-\mu)}\right),
$$

where $\beta=\frac{1}{T}$ is the inverse temperature, and $\mu$ is the chemical potential. The grand potential is given by the spatial integral

$$
\Psi \equiv \int d x \psi(x)
$$

and the thermodynamic properties [entropy, density, free energy, ...] of the system can be derived from $\Psi$ 32]. All information concerning the condensate $\Delta(x)$ is contained in the local density of states $\rho(x ; E)$, and hence in the resolvent $R(x ; E)$. 
For a one-dimensional system [as is being studied here], the resolvent can be expressed in Wronski form, using two independent solutions of $H \psi=E \psi$. By elementary arguments [3, 21, 29, 33], this construction implies that $R(x ; E)$ must satisfy a $2 \times 2$ matrix first-order differential equation, known as the Dikii-Eilenberger equation:

$$
\frac{\partial}{\partial x} R(x ; E) \sigma_{3}=i\left[\left(\begin{array}{cc}
E & -\Delta(x) \\
\Delta^{*}(x) & -E
\end{array}\right), R(x ; E) \sigma_{3}\right] .
$$

It must also satisfy reality and normalization conditions [expressed here for $E$ not in the spectrum, with suitable extensions to other energy branches]:

$$
\operatorname{det} R=-\frac{1}{4} \quad, \quad R^{\dagger}=R
$$

For example, if $\Delta=M$ is a constant, then we have the familiar solution

$$
R(x ; E)=\frac{1}{2 \sqrt{M^{2}-E^{2}}}\left(\begin{array}{cc}
E & M \\
M & E
\end{array}\right)
$$

and it is straightforward to check that this resolvent satisfies the Dikii-Eilenberger equation (1.7).

For non-trivial $\Delta(x)$ it is not so easy to find the exact resolvent, so various expansions have been developed. One such expansion is based on a large $E$ asymtotic expansion of the resolvent:

$$
R(x ; E)=\frac{1}{2} \sum_{n=0}^{\infty} \frac{\hat{r}_{n}(x)}{E^{n}} .
$$

The energy integrals in (1.5) and (1.6) can now be done, leading to an expansion of the grand potential density and the grand potential

$$
\begin{aligned}
\psi(x) & =\sum_{n=0}^{\infty} \alpha_{n}(T, \mu) \hat{g}_{n}(x), \\
\Psi & =\sum_{n=0}^{\infty} \alpha_{n}(T, \mu) \hat{G}_{n} \quad, \quad \hat{G}_{n} \equiv \int d x \hat{g}_{n}(x) .
\end{aligned}
$$

This expansion generalizes a result of Perelomov 34 for the grand potential of systems with Hamiltonians of Schrödinger form, rather than of Dirac form, and which is related in turn to the KdV hierarchy. On the other hand, we show in the next Section that the Dirac case is naturally related to the AKNS hierarchy. The $T$ and $\mu$ dependent coefficients $\alpha_{n}(T, \mu)$ are known functions [2, 3]. The $\hat{g}_{n}(x)$ come from the diagonal elements of the $2 \times 2$ matrices $\hat{r}_{n}(x)$ in the resolvent expansion (1.10):

$$
\hat{r}_{n}(x)=\left(\begin{array}{cc}
\hat{g}_{n}(x) & \hat{f}_{n-1}(x) \\
\hat{f}_{n-1}^{*}(x) & \hat{g}_{n}(x)
\end{array}\right) .
$$

Substituting the expansion (1.10) into the Dikii-Eilenberger equation (1.7), we obtain the simple recursion equations

$$
\begin{aligned}
\hat{f}_{n} & =-\frac{i}{2} \hat{f}_{n-1}^{\prime}+\Delta \hat{g}_{n}, \\
\hat{g}_{n}^{\prime} & =i\left(\Delta^{*} \hat{f}_{n-1}-\Delta \hat{f}_{n-1}^{*}\right) \\
\hat{g}_{0} & =1 \\
\hat{f}_{-1} & =0
\end{aligned}
$$

The initial values follow from the $\Delta=M$ (constant) case above. It is simple to generate these terms recursively. The first few off-diagonal terms are:

$$
\begin{aligned}
\hat{f}_{-1} & =0 \\
\hat{f}_{0} & =\Delta, \\
\hat{f}_{1} & =-\frac{i}{2} \Delta^{\prime}
\end{aligned}
$$




$$
\begin{aligned}
\hat{f}_{2} & =-\frac{1}{4}\left(\Delta^{\prime \prime}-2|\Delta|^{2} \Delta\right) \\
\hat{f}_{3} & =\frac{i}{8}\left(\Delta^{\prime \prime \prime}-6|\Delta|^{2} \Delta^{\prime}\right), \\
\hat{f}_{4} & =\frac{1}{16}\left(\Delta^{(\mathrm{iv})}-8|\Delta|^{2} \Delta^{\prime \prime}-2 \Delta^{2} \Delta^{* \prime \prime}-4\left|\Delta^{\prime}\right|^{2} \Delta-6 \Delta^{*} \Delta^{\prime 2}+6|\Delta|^{4} \Delta\right), \\
\hat{f}_{5} & =-\frac{i}{32}\left(\Delta^{(\mathrm{v})}-10|\Delta|^{2} \Delta^{\prime \prime \prime}-10\left(\Delta \Delta^{* \prime}+2 \Delta^{*} \Delta^{\prime}\right) \Delta^{\prime \prime}-10 \Delta \Delta^{\prime} \Delta^{* \prime \prime}-10 \Delta^{* \prime} \Delta^{\prime 2}+30|\Delta|^{4} \Delta^{\prime}\right), \\
& \vdots
\end{aligned}
$$

The first few diagonal terms are:

$$
\begin{aligned}
\hat{g}_{0} & =1 \\
\hat{g}_{1} & =0 \\
\hat{g}_{2} & =\frac{1}{2}|\Delta|^{2}, \\
\hat{g}_{3} & =\frac{i}{4}\left(\Delta \Delta^{\prime *}-\Delta^{\prime} \Delta^{*}\right), \\
\hat{g}_{4} & =\frac{1}{8}\left(3|\Delta|^{4}+3\left|\Delta^{\prime}\right|^{2}-\left(|\Delta|^{2}\right)^{\prime \prime}\right), \\
\hat{g}_{5} & =\frac{i}{16}\left(\Delta^{\prime \prime \prime} \Delta^{*}-\Delta \Delta^{* \prime \prime \prime}+\Delta^{\prime} \Delta^{* \prime \prime}-\Delta^{\prime \prime} \Delta^{* \prime}+6|\Delta|^{2}\left(\Delta^{* \prime} \Delta-\Delta^{\prime} \Delta^{*}\right)\right) \quad, \\
\hat{g}_{6} & =\frac{1}{32}\left(\Delta^{(\mathrm{iv})} \Delta^{*}+\Delta^{*(\mathrm{iv})} \Delta-\left(\left|\Delta^{\prime}\right|^{2}\right)^{\prime \prime}+3\left|\Delta^{\prime \prime}\right|^{2}-10|\Delta|^{2}\left(\Delta^{\prime \prime} \Delta^{*}+\Delta^{* \prime \prime} \Delta\right)-5\left(\Delta^{* 2} \Delta^{\prime 2}+\Delta^{2} \Delta^{* \prime 2}\right)+10|\Delta|^{6}\right) \\
& =\frac{1}{32}\left(\left(|\Delta|^{2}\right)^{(\mathrm{iv})}-5\left(\left|\Delta^{\prime}\right|^{2}\right)^{\prime \prime}+5\left|\Delta^{\prime \prime}\right|^{2}-10|\Delta|^{2}\left(\Delta^{\prime \prime} \Delta^{*}+\Delta^{* \prime \prime} \Delta\right)-5\left(\Delta^{* 2} \Delta^{\prime 2}+\Delta^{2} \Delta^{* \prime 2}\right)+10|\Delta|^{6}\right), \\
& \vdots
\end{aligned}
$$

Note that in principle there are integration constants appearing in the $\hat{g}_{n}$, but these all vanish by virtue of the normalization condition (1.8).

These recursively generated quantities $\hat{f}_{n}$ and $\hat{g}_{n}$ have many remarkable properties, as is discussed in this paper. For example, the functional variation of $\hat{G}_{n}$ is related to $\hat{f}_{n-2}$ as:

$$
\frac{\delta \hat{G}_{n}}{\delta \Delta^{*}(x)}=\frac{(n-1)}{2} \hat{f}_{n-2}(x)
$$

The physical reason for this remarkable relation is the following. The variation of the grand potential (1.6) can clearly be expressed as

$$
\frac{\delta \Psi}{\delta \Delta^{*}(x)}=\sum_{n=0}^{\infty} \alpha_{n}(T, \mu) \frac{\delta \hat{G}_{n}}{\delta \Delta^{*}(x)} .
$$

On the other hand, the grand potential (1.6) can alternatively be written [3], including the thermodynamical Fermi factor in the energy trace, as

$$
\Psi=\int \frac{d E}{2 \pi} \frac{1}{e^{\beta(E-\mu)}+1} \operatorname{Tr}_{D, x} \ln \left[\gamma^{0}(E-H)\right]
$$

We choose to work in the Dirac gamma matrix representation

$$
\gamma^{0}=\sigma_{1} \equiv\left(\begin{array}{cc}
0 & 1 \\
1 & 0
\end{array}\right) \quad, \quad \gamma^{1}=-i \sigma_{2} \equiv\left(\begin{array}{cc}
0 & -1 \\
1 & 0
\end{array}\right) \quad, \quad \gamma^{5}=\sigma_{3} \equiv\left(\begin{array}{cc}
1 & 0 \\
0 & -1
\end{array}\right) \quad .
$$

Then the BdG Hamiltonian $H$ can be expressed as

$$
H=-i \gamma^{5} \frac{d}{d x}+\gamma^{0}\left[\frac{1}{2}\left(1-\gamma^{5}\right) \Delta(x)+\frac{1}{2}\left(1+\gamma^{5}\right) \Delta^{*}(x)\right] .
$$


Therefore, the variation of the grand potential with respect to $\Delta^{*}$ can be written as

$$
\begin{aligned}
\frac{\delta \Psi}{\delta \Delta^{*}(x)} & =\int \frac{d E}{2 \pi} \frac{1}{e^{\beta(E-\mu)}+1} \operatorname{tr}_{D}\left[\gamma^{0}\left(\mathbf{1}+\gamma^{5}\right) R(x ; E)\right] \\
& =\sum_{n=2}^{\infty} \alpha_{n}(T, \mu) \frac{(n-1)}{2} \hat{f}_{n-2}(x)
\end{aligned}
$$

Comparing (1.21) and (1.25) we find the non-trivial relation (1.20).

\section{AKNS HIERARCHY, FINITE-GAP SYSTEMS AND HIDDEN NONLINEAR SUPERSYMMETRY}

The resolvent expansion functions $\hat{g}_{n}(x)$ and $\hat{f}_{n}(x)$ found in the previous section form the backbone of the (stationary, defocussing) AKNS hierarchy [29, 30, 31, 35], a hierarchy of integrable nonlinear differential equations. The idea behind the integrability of the AKNS hierarchy can be expressed in terms of a Lax pair [36], $S$ and $H$, such that

$$
[S, H]=0
$$

There exists an infinite number of such $S$ commuting with $H$. This is a statement of the integrability of the AKNS system, and also of the BdG Hamiltonian. The Gorkov resolvent $R(x ; E)$ is the generating function for this infinite sequence of conserved quantities. To see this explicitly, define the matrix differential operator of order $(N+1)$ by the following polynomial expansion:

$$
\hat{S}_{N+1}=i \sum_{k=0}^{N+1} \hat{r}_{N+1-k}(x) \sigma_{3} H^{k} \quad, \quad N \in \mathbb{N}_{0}
$$

Here the $\hat{r}_{k}$ are the same quantities that appear in the expansion (1.10) of the Gorkov resolvent. Then the recursion relations (1.17) imply that

$$
\left[\hat{r}_{N+1-k} \sigma_{3}, H\right]=2\left(\begin{array}{cc}
0 & \hat{f}_{N+1-k} \\
-\hat{f}_{N+1-k}^{*} & 0
\end{array}\right)-2\left(\begin{array}{cc}
0 & \hat{f}_{N-k} \\
-\hat{f}_{N-k}^{*} & 0
\end{array}\right) H
$$

This then implies that

$$
\left[\hat{S}_{N+1}, H\right]=\left(\begin{array}{cc}
0 & 2 i \hat{f}_{N+1}(x) \\
-2 i \hat{f}_{N+1}^{*}(x) & 0
\end{array}\right)
$$

The AKNS hierarchy is defined by considering finite linear combinations of the $\hat{S}_{k}$, with coefficients $c_{k}$ :

$$
S_{N+1} \equiv \sum_{k=0}^{N+1} c_{N+1-k} \hat{S}_{k}
$$

Then the $N^{\text {th }}$ equation of the AKNS hierarchy is $\left[S_{N+1}, H\right]=0$, which can equivalently be written as

$$
\sum_{k=0}^{N+1} c_{N+1-k} \hat{f}_{k}=0
$$

For example, the first few equations in the hierarchy are

$$
\begin{aligned}
\mathrm{AKNS}_{0}: & -\frac{i}{2} \Delta^{\prime}+c_{1} \Delta=0, \\
\mathrm{AKNS}_{1}: & -\frac{1}{4}\left(\Delta^{\prime \prime}-2|\Delta|^{2} \Delta\right)-c_{1} \frac{i}{2} \Delta^{\prime}+c_{2} \Delta=0, \\
\mathrm{AKNS}_{2}: & \frac{i}{8}\left(\Delta^{\prime \prime \prime}-6|\Delta|^{2} \Delta^{\prime}\right)-c_{1} \frac{1}{4}\left(\Delta^{\prime \prime}-2|\Delta|^{2} \Delta\right)-c_{2} \frac{i}{2} \Delta^{\prime}+c_{3} \Delta=0 \\
\mathrm{AKNS}_{3}: & \frac{1}{16}\left(\Delta^{(\mathrm{iv})}-8|\Delta|^{2} \Delta^{\prime \prime}-2 \Delta^{2} \Delta^{* \prime \prime}-4\left|\Delta^{\prime}\right|^{2} \Delta-6 \Delta^{*} \Delta^{\prime 2}+6|\Delta|^{4} \Delta\right) \\
& +c_{1} \frac{i}{8}\left(\Delta^{\prime \prime \prime}-6|\Delta|^{2} \Delta^{\prime}\right)-c_{2} \frac{1}{4}\left(\Delta^{\prime \prime}-2|\Delta|^{2} \Delta\right)-c_{3} \frac{i}{2} \Delta^{\prime}+c_{4} \Delta=0
\end{aligned}
$$


The $N=1$ case, $\mathrm{AKNS}_{1}$, is the original AKNS equation, which can also be called the complex nonlinear Schrödinger equation. Another way to see the relation between the Gorkov resolvent and the AKNS hierarchy is to realize that the Dikii-Eilenberger equation is a statement of the zero-curvature representation of the AKNS hierarchy [29, 30].

From a physical standpoint, the AKNS equations arise naturally in a Ginzburg-Landau approach to the BdG system, in which one makes an approximate expansion of the grand potential (1.6) to some finite order, $N+1$ :

$$
\Psi_{\text {Ginzburg-Landau }}=\sum_{k=0}^{N+1} \alpha_{k}(T, \mu) \hat{G}_{k} .
$$

Then the Ginzburg-Landau equation, $\frac{\delta \Psi}{\delta \Delta^{*}(x)}=0$, to this order is precisely an element of the AKNS hierarchy:

$$
\sum_{k=0}^{N+1} \alpha_{k}(T, \mu) \frac{(k-1)}{2} \hat{f}_{k-2}=0
$$

with the coefficients expressed in terms of the real-valued functions $\alpha_{k}(T, \mu)$. Recall that $\hat{f}_{-2}=\hat{f}_{-1}=0$.

As already mentioned, the elements of the AKNS hierarchy have some remarkable properties, and because of the relation between the BdG resolvent and the AKNS hierarchy, these properties turn out to be very useful in solving physical problems based on the BdG Hamiltonian.

For example, suppose $\Delta$ satisfies the $(N+1)^{\text {th }}$ order differential equation $\mathrm{AKNS}_{N}$. It automatically follows from the recursion relations (1.17) that $\Delta$ satisfies all the higher equations $\mathrm{AKNS}_{N+k}$, for $k=1,2, \ldots$, with a suitable choice of the coefficients $c_{k}$. This means that there is an infinite number of conserved quantities for any member of the AKNS hierarchy. Physically, this has the important implication that it is possible to make a consistent polynomial ansatz for the Gorkov resolvent in order to solve the Dikii-Eilenberger equation (1.7), as was done in [3]. Thus, if we make the polynomial ansatz for $R(x ; E)$ :

$$
R(x ; E)=\sum_{n=0}^{N} \beta_{n}(E)\left(\begin{array}{cc}
\hat{g}_{n}(x) & \hat{f}_{n-1}(x) \\
\hat{f}_{n-1}^{*}(x) & \hat{g}_{n}(x)
\end{array}\right)
$$

then $R(x ; E)$ satisfies the Dikii-Eilenberger equation (1.7) provided $\Delta(x)$ satisfies the $\mathrm{AKNS}_{N-1}$ equation. Therefore, given a solution of $\mathrm{AKNS}_{N-1}$, the exact resolvent can be written in a simple polynomial form (2.13), with the coefficients $\beta_{n}(E)$ determined from simple algebraic relations. This fact was used in [3] to solve the gap equation in the $\mathrm{NJL}_{2}$ model, using $N=2$ in which case the condensate function $\Delta(x)$ had to satisfy the $\mathrm{AKNS}_{1}$ equation, otherwise known as the (complex) nonlinear Schrödinger equation. In fact, this polynomial form of the resolvent of the BdG Hamiltonian generalizes an analogous property of Schrödinger Hamiltonians. For example, it was known already to Hermite that for the Lamé potential, $V(x)=N(N+1) \nu \operatorname{sn}^{2}(x)$, with $N$ integer, the resolvent can be written as a polynomial of degree $N$ in $V(x)$ [13].

Next we connect this discussion to the known finite gap solutions of the AKNS hierarchy [31, 35]. First, we discuss the spectral properties of the explicit finite gap solutions, and then explain their relation to nonlinear quantum mechanical supersymmetry. The remaining sections will discuss details of explicit cases where $\Delta$ is trigonometric (non-periodic case) or elliptic (periodic case).

\section{A. Finite gap solutions to the AKNS hierarchy}

When $\Delta$ satisfies an equation from the AKNS hierarchy, the spectrum of the system (1.1) acquires a finite-gap nature [31, 37, 38]. Suppose we have a complex periodic $\Delta$ that obeys the $\mathrm{AKNS}_{N}$ equation [this picture can be extended for the case of a quasi-periodic $\Delta$; see Section IV below]. The spectrum of (1.1) is composed then of $N$ valence bands, $\left[E_{2 k-1}, E_{2 k}\right], k=1, \ldots, N$, and by two conduction bands, $\left(-\infty, E_{0}\right]$ and $\left[E_{2 N+1}, \infty\right)$, separated by $N+1$ energy gaps,

$$
\sigma(H)=\left(-\infty, E_{0}\right] \cup\left[E_{1}, E_{2}\right] \cup \ldots \cup\left[E_{2 N-1}, E_{2 N}\right] \cup\left[E_{2 N+1}, \infty\right) .
$$

The $2 N+2$ energies $E_{k}, k=0, \ldots, 2 N+1$, correspond to singlet band-edge states, which are described either by periodic or by anti-periodic wave functions. The states in the interior of the allowed bands are described by Bloch-Floquet quasi-periodic functions, and any corrresponding energy level is doubly degenerate, corresponding physically to leftand right-going waves. In the infinite period limit, $\Delta$ transforms into a non-periodic function, $N$ valence bands shrink and transform into $N$ bound states; the quasi-periodic states of the conduction bands reduce to scattering states with energies continuously varying in the intervals $\left(-\infty, E_{0}\right]$ and $\left[E_{2 N+1}, \infty\right)$, where all energy levels, except edge values, are doubly degenerate. 


\section{B. Nonlinear quantum mechanical supersymmetry of $\mathrm{AKNS}_{N}$}

A double degeneration of the spectrum, accompanied by a presence of a finite number of singlet states, is a typical property of $\mathcal{N}=2$ nonlinear quantum mechanical supersymmetry [26, 39, 40, 41]. In order to identify its structure for the finite-gap Bogoliubov-de Gennes system, we first describe briefly different realizations of $\mathcal{N}=2$ supersymmetry in Schrödinger systems with a second order Hamiltonian.

Quantum mechanical supersymmetry in its simplest form [42, 43, 44] reflects coherently spectral properties of a composed system, given by superpartner Schrödinger Hamiltonians $\mathcal{H}^{ \pm}=-d^{2} / d x^{2}+V^{ \pm}(x), V^{ \pm}(x)=W^{2}(x) \pm W^{\prime}(x)$. The latter obey intertwining relations of a Darboux transformation [45],

$$
A_{1} \mathcal{H}^{-}=\mathcal{H}^{+} A_{1} \quad, \quad A_{1}^{\dagger} \mathcal{H}^{+}=\mathcal{H}^{-} A_{1}^{\dagger} .
$$

Here $A_{1}=\psi_{0} \frac{d}{d x} \frac{1}{\psi_{0}}=\frac{d}{d x}+W(x)$ is a first order differential operator, whose kernel is a (generically, formal) zero-value eigenstate $\psi_{0}(x)$ of the second order differential operator $\mathcal{H}^{-}$. If $\psi_{0}(x)$ is a nodeless function, the superpotential $W(x)=-\frac{d}{d x} \ln \psi_{0}(x)$ and both superpartner potentials $V^{ \pm}(x)$ are regular. Factorizations $\mathcal{H}^{-}=A_{1}^{\dagger} A_{1}$ and $\mathcal{H}^{+}=$ $A_{1} A_{1}^{\dagger}$ reflect a non-negative nature of the spectra of both subsystems, which in a generic case are almost isospectral by virtue of intertwining relations (2.15). In non-periodic case, when neither of the states $\psi_{0}$ and $1 / \psi_{0}($ the latter being a kernel of the operator $A_{1}^{\dagger}$ ) is physical, i.e. is not a normalizable function, two subsystems have exactly the same positive definite spectrum. If one of the functions $\psi_{0}$ or $1 / \psi_{0}$ is normalizable, it is a singlet zero-energy state of the supersymmetric system $\mathcal{H}=\operatorname{diag}\left(\mathcal{H}^{+}, \mathcal{H}^{-}\right)$. In periodic case both states $\psi_{0}$ and $1 / \psi_{0}$ are physical when they are periodic, and the lowest zero-energy level is doubly degenerate [47].

Intertwining and factorization relations are rewritten equivalently in the form of a linear (Lie) $\mathcal{N}=2$ superalgebra

$$
\left[\mathcal{Q}_{a}, \mathcal{H}\right]=0 \quad, \quad\left\{\mathcal{Q}_{a}, \mathcal{Q}_{b}\right\}=2 \delta_{a b} \mathcal{H} \quad,
$$

generated by the matrix Hamitlonian $\mathcal{H}$ and supercharges

$$
\mathcal{Q}_{1}=i\left(\begin{array}{cc}
0 & A_{1} \\
-A_{1}^{\dagger} & 0
\end{array}\right) \quad, \quad \mathcal{Q}_{2}=i \sigma_{3} \mathcal{Q}_{1}
$$

The integral of motion $\Gamma=\sigma_{3}$, with $\Gamma^{2}=1$, plays here the role of a $\mathbb{Z}_{2}$-grading operator,

$$
[\Gamma, \mathcal{H}]=0 \quad, \quad\left\{\Gamma, Q_{a}\right\}=0 \quad
$$

Eq. (2.15) is generalized for the case of a Crum-Darboux transformation [45] generated by an intertwining operator $A_{n}$ of order $n>1$, which annihilates $n$ linear independent (generically, formal) eigenstates of $\mathcal{H}^{-}$. The partner Hamiltonians are related by $\mathcal{H}^{+}=\mathcal{H}^{-}-2\left(\ln \mathcal{W}_{n}\left(\psi_{1}, \ldots, \psi_{n}\right)\right)^{\prime \prime}$, where $\mathcal{W}_{n}$ is the Wronskian of the states of the kernel of $A_{n}$. If $\mathcal{W}_{n}(x)$ is a nodeless function, the partner Hamiltonians $\mathcal{H}^{ \pm}$have the same regularity properties. Intertwining relations are reformulated, again, in the form of zero commutators of the supercharges $\mathcal{Q}_{a}$, constructed in the form (2.17) with $A_{1}$ substituted by $A_{n}$, with matrix Hamiltonian $\mathcal{H}=\operatorname{diag}\left(\mathcal{H}^{+}, \mathcal{H}^{-}\right)$. In correspondence with Burchnall-Chaundy theorem [48, 49], the linear superalgebraic relation (2.16) becomes a nonlinear one,

$$
\left\{\mathcal{Q}_{a}, \mathcal{Q}_{b}\right\}=2 \delta_{a b} P_{n}(\mathcal{H})
$$

where $P_{n}(\mathcal{H})$ is a polynomial of order $n$ in the Hamiltonian. For such a non-linear supersymmetry, of order $n$, the operator $\Gamma=\sigma_{3}$ is again a $\mathbb{Z}_{2}$-grading operator. The spectra of the two superpartner Schrödinger Hamiltonians $\mathcal{H}^{ \pm}$ may differ in $0 \leq l \leq n$ energy levels.

Some non-extended systems with second order Hamiltonian are characterized by a hidden supersymmetry of a bosonized nature [39, 40, 41]. In this case $\mathcal{H}^{+}=\mathcal{H}^{-}$, and the intertwining relation reduces to the commutativity of $\mathcal{H}^{-}$with a Hermitian differential operator which itself is identified as one of the supercharges, $\mathcal{Q}_{1}=\mathcal{Z}$. Another supercharge is $\mathcal{Q}_{2}=i \Gamma \mathcal{Q}_{1}$, where a $\mathbb{Z}_{2}$-grading operator $\Gamma$ is now a parity (reflection), or a twisted parity operator [40]. A bosonized supersymmetry of a nonlinear form appears, in particular, in finite-gap periodic and reflectionless nonperiodic second order systems, in which a nontrivial operator of a Lax pair is identified as supercharge $Q_{1}$ annihilating all the singlet energy eigenstates.

Corresponding systems extended by means of a Darboux or a Crum-Darboux transformation are described by a more rich, tri-supersymmetric structure [50, 51, 52] . The latter admits three alternative choices for $\mathbb{Z}_{2}$-grading operator, $\Gamma=\sigma_{3}, \mathcal{R}$, or $\mathcal{R} \sigma_{3}$, where $\mathcal{R}$ is a parity operator, and will be discussed in more detail in the next sections.

For any type of supersymmetry described above, infinitesimal supersymmetric transformations generated by a supercharge $\mathcal{Q}$ can be presented in a form

$$
\delta \mathcal{X}=[\delta \alpha \mathcal{Q}, \mathcal{X}] \quad, \quad \delta \alpha=\xi \Gamma \quad,
$$


where $\delta \alpha$ is a composition of a Grassmann parameter $\xi$ and of a $\mathbb{Z}_{2}$-grading operator $\Gamma$.

For the Bogoliubov-de Gennes system, Hamiltonian (1.1) is a $2 \times 2$ matrix first order differential operator. When (in a generic case complex) the function $\Delta$ satisfies the $\mathrm{AKNS}_{N}$ equation, the system displays a non-linear supersymmetry of order $(N+1)$. To identify its structure, we consider the $2 \times 2$ matrix operator $S_{N+1}$ in (2.5) as one of the supercharges, which annihilates the whole set of $(2 N+2)$ band edge states,

$$
S_{N+1} \psi_{k}=0 \quad, \quad H \psi_{k}=E_{k} \psi_{k} \quad, \quad k=0,1 \ldots, 2 n+1 \quad .
$$

Using a matrix analogue of the Burchnall-Chaundy theorem, one finds that the square of $S_{N+1}$ produces a spectral polynomial of degree $(2 N+2)$ [69],

$$
S_{N+1}^{2}=P_{2 N+2}(H)=\prod_{k=0}^{2 N+1}\left(H-E_{k}\right),
$$

associated with the hyperelliptic curve of genus $N$. We will show that for any particular choice of the function $\Delta$ satisfying the $\mathrm{AKNS}_{N}$ equation, there exists an integral $\Gamma$, unitary equivalent to a nonlocal operator $\mathcal{R} \sigma_{3}$, with the properties

$$
[H, \Gamma]=0 \quad, \quad\left\{S_{N+1}, \Gamma\right\}=0 \quad, \quad \Gamma^{2}=1 .
$$

Identifying integral $\Gamma$ as a $\mathbb{Z}_{2}$-grading operator, we treat $S_{N+1}$ as one of the supercharges, $\mathcal{Q}_{1}=S_{N+1}$, and define another supercharge in the usual way, $\mathcal{Q}_{2}=i \Gamma \mathcal{Q}_{1}$. As a result we find that the system is described by $\mathcal{N}=2$ nonlinear supersymmetry,

$$
\left[\mathcal{Q}_{a}, H\right]=0 \quad, \quad\left\{\mathcal{Q}_{a}, \mathcal{Q}_{b}\right\}=2 \delta_{a b} P_{2 N+2}(H)
$$

The supersymmetric transformations are given by Eq. (2.20).

Notice that the described general structure of nonlinear supersymmetry of the finite-gap Bogoliubov-de Gennes system is similar to the hidden bosonized supersymmetry of a finite-gap Schrödinger system [40, 41]. In both cases nontrivial integrals annihilate all the singlet states, all the other physical states are organized in energy doublets, and the corresponding $\mathbb{Z}_{2}$-grading operators are nonlocal.

\section{REAL DEFOCUSING HIERARCHY NS + AND TRI-SUPERSYMMETRY}

In this Section we consider the real case $\Delta=\Delta^{*}$ of the BdG system 1.1. As it follows from a unitary transformation corresponding to a global chiral rotation with constant parameter $\varphi$

$$
U(\varphi) H(\Delta) U^{-1}(\varphi)=H(\tilde{\Delta}) \quad, \quad U(\varphi)=e^{\frac{i}{2} \varphi \gamma^{5}}, \quad \tilde{\Delta}=e^{i \varphi} \Delta
$$

a real case of $\Delta$ can be understood modulo $U(1)$.

When $\Delta=\Delta^{*}$, the Hamiltonian anticommutes with $i \gamma^{1}=\sigma_{2}$. As a consequence, the spectrum is symmetric with respect to the energy reflection $E \rightarrow-E$. Then the energies of singlet band-edge states (2.14) obey a symmetry

$$
E_{2 N+1-i}=-E_{i} \quad, \quad i=0, . ., N \quad .
$$

For the following discussion it is convenient to present Hamiltonian (1.1) in another form by applying a unitary transformation generated by a matrix

$$
\mathcal{U}=\frac{1}{2}\left(\begin{array}{cc}
1+i & 1-i \\
-1-i & 1-i
\end{array}\right)
$$

This amounts to a change of Dirac representation from (1.23) to

$$
\gamma^{0}=-\sigma_{2} \quad, \quad \gamma^{1}=-i \sigma_{3} \quad, \quad \gamma^{5}=-\sigma_{1} .
$$

The BdG Hamiltonian (1.24) with real $\Delta$ takes therefore a form

$$
H_{\mathbb{R}}=i\left(\begin{array}{cc}
0 & \frac{d}{d x}+\Delta(x) \\
\frac{d}{d x}-\Delta(x) & 0
\end{array}\right) .
$$


This coincides with one of the matrix supercharges (2.17) of a supersymmetric quantum mechanicanical system [42] described by a matrix Schrödinger Hamiltonian $\mathcal{H}=H_{\mathbb{R}}^{2}$, for which $\Delta=W(x)$ is identified as the superpotential, and supersymmetric partner potentials are given by

$$
V^{ \pm}(x)=\Delta^{2} \pm \Delta^{\prime}
$$

In accordance with (1.23) [and also (4.6) below], the operator $i \gamma^{1}$ underlying the energy reflection symmetry of the real BdG system corresponds to the grading operator $\Gamma=\sigma_{3}$ for the associated superextended Schrödinger system.

If $\Delta$ satisfies the $\mathrm{AKNS}_{N}$ equation, the number of gaps modulo 2 in the spectrum of the periodic BdG system (or, of bound states in the non-periodic limit) depends on the properties of periodicity (normalizability) of the functions

$$
\Psi_{0}^{ \pm}(x)=e^{ \pm \int_{0}^{x} \Delta(y) d y} u^{ \pm},
$$

where $u^{+}=(1,0)^{t}, u^{-}=(0,1)^{t}$, and $t$ means transposition. Namely, if $N=2 m-1, m \in \mathbb{N}$, and both functions (3.7) are periodic (or, one of these functions is normalizable), they describe two (or, one in a non-periodic case) zero energy physical states of the system (3.5) with even number of gaps in a spectrum. For $N=2 m, m \in \mathbb{N}$, states (3.7) are not periodic (normalizable in the non-periodic limit), and level $E=0$ does not belong to the spectrum characterized by odd number of gaps.

From the viewpoint of the associated super-extended Schrödinger Hamiltonian

$$
\mathcal{H}=H_{\mathbb{R}}^{2},
$$

the cases $N=2 m-1$ and $N=2 m$ correspond to finite-gap systems with exact and broken $\mathcal{N}=2$ supersymmetries, respectively.

When real $\Delta$ satisfies an AKNS equation, the equality (3.8), that connects the BdG and extended Schrödinger Hamiltonians, reflects an intimate relation between the AKNS and Korteweg- de Vries (KdV) hierarchies (see for example [31]). This is just because in this case the Schrödinger potentials $V^{ \pm}$satisfy equations of the corresponding KdV stationary hierarchies. Therefore, each component $\mathcal{H}^{ \pm}$of the diagonal Schrödinger Hamiltonian $\mathcal{H}$ has a nontrivial integral of motion in the form of a differential operator $\mathcal{Z}^{ \pm}$from the corresponding Lax pair $\left(\mathcal{H}^{ \pm}, \mathcal{Z}^{ \pm}\right)$of the $\mathrm{KdV}$ hierarchy. The diagonal matrix operator $\mathcal{Z}=\operatorname{diag}\left(\mathcal{Z}^{+}, \mathcal{Z}^{-}\right)$commutes with the matrix Hamiltonian, $[\mathcal{H}, \mathcal{Z}]=0$, and leads to existence of a more rich, tri-supersymmetric structure [51, 52], which will be discussed in more detail below. The systems with exact and broken $\mathcal{N}=2$ supersymmetry reveal the tri-supersymmetric structure in different ways, and these two cases will be considered separately.

\section{A. Exact $\mathcal{N}=2$ supersymmetry and tri-supersymmetry in the light of the $\mathrm{nS}_{+}$hierarchy}

Let us start with the case of unbroken $\mathcal{N}=2$ supersymmetry, and consider the BdG system (3.5) given by a real odd function $\Delta(x)$,

$$
\Delta(-x)=-\Delta(x)
$$

satisfying the $(2 m)^{\text {th }}$ order equation $\mathrm{AKNS}_{2 m-1}, m \in \mathbb{N}$. Since Hamiltonian $H_{\mathbb{R}}$ anticommutes with both $\sigma_{3}$ and $\mathcal{R}$, the operator $\mathcal{R} \sigma_{3}$ is a non-local integral of motion, $\left[H_{\mathbb{R}}, \mathcal{R} \sigma_{3}\right]=0$. The stationary $2 m-1$-th order AKNS hierarchy is described by the Lax pair $\left(H_{\mathbb{R}}, S_{2 m}\right)$, where $S_{2 m}$ is a non-trivial integral of motion, $\left[H_{\mathbb{R}}, S_{2 m}\right]=0$. In the present case it anticommutes with the trivial integral $\mathcal{R} \sigma_{3},\left\{S_{2 m}, \mathcal{R} \sigma_{3}\right\}=0$. We can treat $\mathcal{R} \sigma_{3}$ as the grading operator, and $S_{2 m}$ as a non-linear supercharge for the BdG Hamiltonian in accordance with relations (2.24) and (2.23), where $N=2 m-1$.

Because of the presence of the nontrivial integral $S_{2 m}$ in the BdG system, the associated extended Schrödinger system with parity-even Hamiltonian $\mathcal{H}$ is characterized by a more rich than $\mathcal{N}=2$ supersymmetry structure. Indeed, relation (3.8) implies that the matrix operator $S_{2 m}$ is also the integral of motion for the associated Schrödinger system. Denoting $H_{\mathbb{R}}=\mathcal{X}$ and $S_{2 m}=\mathcal{Y}$, we have

$$
[\mathcal{H}, \mathcal{X}]=[\mathcal{H}, \mathcal{Y}]=0 \quad, \quad[\mathcal{X}, \mathcal{Y}]=0,
$$

i.e. $\mathcal{X}$ and $\mathcal{Y}$ are commuting parity-odd and parity-even integrals, respectively. As we noted above, the extended Schrödinger system $\mathcal{H}$ is characterized also by the diagonal higher order matrix integral $\mathcal{Z}$. Its relation with the original BdG system can easily be established. Consider the next after $S_{2 m}$ integral $S_{2 m+1}$ from the infinite set of integrals of the corresponding AKNS hierarchy. For the BdG Hamiltonian $H_{\mathbb{R}}$, operator $S_{2 m+1}$ appears as a trivial composite integral,

$$
S_{2 m+1}=H_{\mathbb{R}} S_{2 m}=S_{2 m} H_{\mathbb{R}}
$$


For the associated extended Schrödinger system, this is exactly the nontrivial diagonal parity-odd integral, $S_{2 m+1}=\mathcal{Z}$,

$$
\mathcal{Z}=\mathcal{X} \mathcal{Y}=\mathcal{Y} \mathcal{X}
$$

Then relations (3.10) can be supplied by

$$
[\mathcal{H}, \mathcal{Z}]=0 \quad, \quad[\mathcal{Z}, \mathcal{X}]=[\mathcal{Z}, \mathcal{Y}]=0
$$

Operators $\mathcal{X}, \mathcal{Y}$ and $\mathcal{Z}$ constitute a part of nontrivial integrals for the Schrödinger system. Hamiltonian $\mathcal{H}$ commutes also with mutually commuting trivial integrals $\sigma_{3}, \mathcal{R}$ and $\mathcal{R} \sigma_{3}$, and their products with $\mathcal{X}, \mathcal{Y}$ and $\mathcal{Z}$ produce other nontrivial integrals of motion. All these operators together generate the tri-supersymmetry of the second order finitegap system $\mathcal{H}$. The resulting complete nonlinear superalgebraic structure depends on the identification of one of the trivial integrals, $\sigma_{3}, \mathcal{R}$, or $\mathcal{R} \sigma_{3}$, as the grading operator. For any of the three possible choices, exactly one of the nontrivial integrals $\mathcal{X}, \mathcal{Y}$, or $\mathcal{Z}$ is identified as $\mathbb{Z}_{2}$-even operator, which plays the role of the central charge of the superalgebra, while other two are treated as $\mathbb{Z}_{2}$-odd supercharges. This is in contrast with the supersymmetric structure of the BdG system $H_{\mathbb{R}}$, for which the only unitary operator $\mathcal{R} \sigma_{3}$ can be chosen as the grading operator. For the details on the tri-supersymmetry we refer the reader to [51, 52].

Next we discuss some concrete examples of solutions and the associated explicit form of the nonlinear supersymmetric structure for the real $\mathrm{AKNS}_{N}$ systems with $N=2 m-1, m \in \mathbb{N}$.

\section{Real periodic solution of the $(2 m-1)$ th $n S_{+}$equation}

Consider a periodic solution of the $(2 m-1)^{\text {th }} \mathrm{nS}_{+}$equation [70],

$$
\Delta=m \nu \frac{\operatorname{sn}(x, \nu) \operatorname{cn}(x, \nu)}{\operatorname{dn}(x, \nu)},
$$

where $m \in \mathbb{N}$, and $\operatorname{sn}(x, \nu)=\operatorname{sn} x, \operatorname{cn}(x, \nu)=\operatorname{cn} x$ and $\operatorname{dn}(x, \nu)=\operatorname{dn} x$ are the Jacobi elliptic functions of elliptic parameter $\nu$, with $0<\nu<1$ [53]. The functions $\operatorname{cn} x$ and $\operatorname{dn} x$ are even, while $\operatorname{sn} x$ is odd, and therefore (3.14) is an odd function. In this case the Hamiltonian (3.5) takes the form of an odd operator

$$
H_{\mathbb{R}}=i\left(\begin{array}{cc}
0 & \frac{d}{d x}+m \nu \frac{\operatorname{sn} x \operatorname{cn} x}{\operatorname{dn} x} \\
\frac{d}{d x}-m \nu \frac{\operatorname{sn} x \operatorname{cn} x}{\operatorname{dn} x} & 0
\end{array}\right) .
$$

Therefore, the system is characterized by a nonlocal integral of motion

$$
\Gamma=\mathcal{R} \sigma_{3}
$$

Hamiltonian (3.15) has a real period $T=2 \mathbf{K}(\nu), H_{\mathbb{R}}(x)=H_{\mathbb{R}}(x+T)$, where $\mathbf{K}(\nu)$ is the complete elliptic integral of the first kind. This system has $4 m$ band edge-states of non-degenerated energies $E_{i}, i=0, \ldots, 4 m-1$, distributed symmetrically [see Figure 1],

$$
E_{4 m-1-i}=-E_{i} \quad, \quad i=0, . ., 2 m \quad,
$$

with respect to the doubly degenerate zero energy level, which is in the middle of the central allowed band. All the band-edge singlet states are anti-periodic, their period is $4 \mathbf{K}(\nu)$.

With $\Delta$ satisfying the $(2 m-1)^{\text {th }} \mathrm{nS}_{+}$equation, the Hamiltonian (3.15) commutes with a non-trivial integral of motion (2.5), which can be presented in a form

$$
S_{2 m}=\left(\begin{array}{cc}
0 & S_{2 m}^{-} \\
S_{2 m}^{+} & 0
\end{array}\right)
$$

where

$$
S_{2 m}^{-}=\frac{\mathrm{dn}^{m+1} x}{\operatorname{cn}^{2 m+1} x}\left(\frac{\operatorname{cn}^{2} x}{\operatorname{dn} x} \frac{d}{d x}\right)^{2 m} \frac{\operatorname{dn}^{m-1} x}{\operatorname{cn}^{2 m-1} x} \quad, \quad S_{2 m}^{+}=\left(S_{2 m}^{-}\right)^{\dagger}
$$

Since $S_{2 m}^{-}$is an even differential operator, (3.18) anti-commutes with the integral (3.16), $\left\{S_{2 m}, \mathcal{R} \sigma_{3}\right\}=0$. Operator (3.16) is identified as a $\mathbb{Z}_{2}$-grading operator, and integrals

$$
\mathcal{Q}_{1}=S_{2 m} \quad, \quad \mathcal{Q}_{2}=i \mathcal{R} \sigma_{3} \mathcal{Q}_{1}
$$




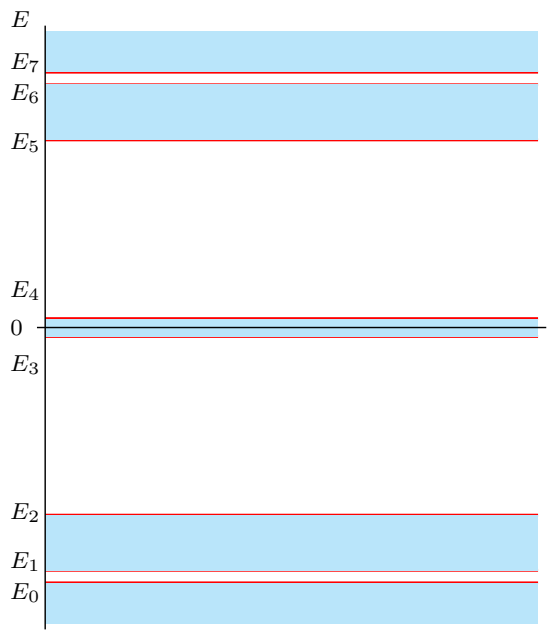

FIG. 1: Spectrum of (3.15) for $m=2$ and with $\nu=0.9$. Red lines are the singlet band edge energies. The black line represents the zero energy level, with respect to which all the spectrum is symmetric.

play the role of the supercharges for the system (3.15). They generate the $\mathcal{N}=2$ polynomial superalgebra

$$
\left[\mathcal{Q}_{a}, H_{\mathbb{R}}\right]=0 \quad, \quad\left\{\mathcal{Q}_{a}, \mathcal{Q}_{b}\right\}=2 \delta_{a b} P_{4 m}\left(H_{\mathbb{R}}\right)
$$

where, in correspondence with (3.17),

$$
P_{4 m}\left(H_{\mathbb{R}}\right)=\prod_{i=0}^{4 m-1}\left(H_{\mathbb{R}}-E_{i}\right)=\prod_{i=0}^{2 m-1}\left(H_{\mathbb{R}}^{2}-E_{i}^{2}\right)
$$

The associated with (3.15) superextended Schrödinger system given by the second order Hamiltonian $\mathcal{H}=H_{\mathbb{R}}^{2}$ describes, in correspondence with Eq. (3.17), the $m$-gap self-isospectral Lamé system [47, 51, 52] with band-edge energies $\tilde{E}_{0}=0, \tilde{E}_{j}=E_{2 m-1+j}^{2}=E_{2 m-j}^{2}>0, j=1, \ldots, 2 m$.

For the BdG Hamiltonian in the simplest case $m=1$, the singlet band-edge states and their energies are given by

$$
\begin{gathered}
\psi_{0}=\left(\begin{array}{c}
i \operatorname{cn}(x+b / 2) \\
-\operatorname{cn}(x-b / 2)
\end{array}\right) \quad, \quad \psi_{1}=\left(\begin{array}{c}
i \sin (x+b / 2) \\
-\operatorname{sn}(x-b / 2)
\end{array}\right), \quad \psi_{2}=\sigma_{3} \psi_{1} \quad, \quad \psi_{3}=\sigma_{3} \psi_{0} \\
H_{\mathbb{R}} \psi_{i}=E_{i} \psi_{i} \quad, \quad E_{0}=-1 \quad, \quad E_{1}=-\sqrt{1-\nu}, \quad E_{2}=-E_{1} \quad, \quad E_{3}=-E_{0}
\end{gathered}
$$

The explicit form of the states in the interior of the allowed bands can be found, for example, in refs. [54].

\section{Real non-periodic solution of the $(2 m-1)$ th $n S_{+}$equation}

Taking the infinite period limit of (3.14), which corresponds to $\nu \rightarrow 1$, we get a non-periodic solution of the $(2 m-1)$ th $\mathrm{nS}_{+}$equation, the familiar kink

$$
\Delta=m \tanh x \quad, \quad m \in \mathbb{N} .
$$

Hamiltonian (3.5) takes a form

$$
H_{\mathbb{R}}=i\left(\begin{array}{cc}
0 & \frac{d}{d x}+m \tanh x \\
\frac{d}{d x}-m \tanh x & 0
\end{array}\right)=i\left(\begin{array}{cc}
0 & \mathcal{D}_{m} \\
\mathcal{D}_{-m} & 0
\end{array}\right),
$$

where the operator $\mathcal{D}_{\lambda}$ satisfies the relations $\mathcal{D}_{-\lambda}=-\mathcal{D}_{\lambda}^{\dagger}$ and $\mathcal{D}_{-\lambda} \mathcal{D}_{\lambda}=\mathcal{D}_{\lambda+1} \mathcal{D}_{-\lambda-1}+2 \lambda+1$.

The central band $\left[E_{2 m-1}, E_{2 m}\right], E_{2 m-1}=-E_{2 m}$, of the periodic system shrinks here into a zero energy level, $\left[E_{2 m-1}, E_{2 m}\right] \rightarrow \mathcal{E}_{m}=0$, and the corresponding band-edge states transform into a single bound state. The other valence bands shrink as well, producing $(2 m-1)$ bound states of non-zero values of energy: $\left[E_{1}, E_{2}\right] \rightarrow \mathcal{E}_{1},\left[E_{3}, E_{4}\right] \rightarrow$ $\mathcal{E}_{2}$, etc. The conduction bands transform into scattering bands with singlet edge energies $\mathcal{E}_{0}<0$ and $\mathcal{E}_{2 m}=-\mathcal{E}_{0}>0$, where $E_{0} \rightarrow \mathcal{E}_{0}, E_{4 m-1}=-E_{0} \rightarrow \mathcal{E}_{2 m}$. 
Defining the higher order differential operators

$$
\mathcal{D}_{-\lambda}^{\beta}=\mathcal{D}_{-\lambda} \mathcal{D}_{-\lambda+1} \ldots \mathcal{D}_{-\lambda+\beta-1} \quad, \quad \beta=2, \ldots, \lambda \quad, \quad \mathcal{D}_{-\lambda}^{0}=1, \quad \mathcal{D}_{-\lambda}^{1}=\mathcal{D}_{-\lambda},
$$

we can write easily the eigenfunctions of (3.26). The $2 m+1$ singlet states acquire a form

$$
\begin{gathered}
\psi_{m, l}^{ \pm}(x)=\left(\begin{array}{c}
\mp i \mathcal{E}_{m-l} \mathcal{D}_{-m+1}^{l-1} \operatorname{sech}^{m-l} x \\
\mathcal{D}_{-m}^{l} \operatorname{sech}^{m-l} x
\end{array}\right) \quad, \quad l=0, \ldots, m \\
H_{\mathbb{R}} \psi_{m, l}^{ \pm}(x)= \pm \mathcal{E}_{m-l} \psi_{m, l}^{ \pm}(x) \quad, \quad \mathcal{E}_{m-l}=\sqrt{m^{2}-(m-l)^{2}}
\end{gathered}
$$

Here $l=m$ corresponds to two singlet edge states of the two symmetric parts of the continuous spectrum, while $l=0, . ., m-1$ give $(2 m-1)$ bound states. For $l=0$, wave functions $\psi_{m, 0}^{+}(x)$ and $\psi_{m, 0}^{-}(x)$ coincide, $\psi_{m, 0}^{+}(x)=$ $\psi_{m, 0}^{-}(x)=\psi_{m, 0}(x)$,

$$
\psi_{m, 0}(x)=\left(\begin{array}{c}
0 \\
\operatorname{sech}^{m} x
\end{array}\right)
$$

This function describes a singlet bound state with energy $\mathcal{E}_{m}=0$ in the middle of the spectrum. The scattering states of the bands $\left(-\infty, \mathcal{E}_{0}\right]$ and $\left[\mathcal{E}_{2 m}, \infty\right)$ have a form

$$
\begin{aligned}
\psi_{m ; \uparrow(\downarrow)}^{ \pm}(x) & =\left(\begin{array}{c}
-(+) i \mathcal{E}_{m ; k} \mathcal{D}_{-m+1}^{m-1} e^{ \pm i k x} \\
\mathcal{D}_{-m}^{m} e^{ \pm i k x}
\end{array}\right), \\
H_{\mathbb{R}} \psi_{m ; \uparrow}^{ \pm}(x)=\mathcal{E}_{m ; k} \psi_{m ; \uparrow}^{ \pm}(x) \quad, \quad H_{\mathbb{R}} \psi_{m ; \downarrow}^{ \pm}(x) & =-\mathcal{E}_{m ; k} \psi_{m ; \downarrow}^{ \pm}(x) \quad, \quad \mathcal{E}_{m: k}=\sqrt{m^{2}+k^{2}},
\end{aligned}
$$

where $k>0$. Boundary value $k=0$ reproduces singlet edge states (3.27) with $l=m$.

The non-trivial integral (3.18) transforms into

$$
S_{2 m}=\left(\begin{array}{cc}
0 & \left(\mathcal{D}_{-m}^{2 m}\right)^{\dagger} \\
\mathcal{D}_{-m}^{2 m} & 0
\end{array}\right)=\left(\begin{array}{cc}
0 & \mathcal{D}_{-m+1} \mathcal{D}_{-m+2} \ldots \mathcal{D}_{m-1} \mathcal{D}_{m} \\
\mathcal{D}_{-m} \mathcal{D}_{-m+1} \ldots \mathcal{D}_{m-2} \mathcal{D}_{m-1} & 0
\end{array}\right)
$$

The supercharges are identified as in (3.20). They annihilate all the $2 m+1$ singlet states. The nonlinear superalgebra is given by the same equation (3.21) as in the periodic case, but with the spectral polynomial operator changed coherently with the bands shrunk,

$$
P_{4 m}\left(H_{\mathbb{R}}\right)=\left(H_{\mathbb{R}}^{2}-\mathcal{E}_{0}^{2}\right)\left(H_{\mathbb{R}}-\mathcal{E}_{m}\right)^{2} \prod_{i=1}^{m-1}\left(H_{\mathbb{R}}^{2}-\mathcal{E}_{i}^{2}\right)^{2}=\left(H_{\mathbb{R}}-\mathcal{E}_{0}\right)\left(H_{\mathbb{R}}-\mathcal{E}_{2 m}\right) \prod_{i=1}^{2 m-1}\left(H_{\mathbb{R}}-\mathcal{E}_{i}\right)^{2}
$$

The relation $S_{2 m}^{2}=P_{4 m}\left(H_{\mathbb{R}}\right)$ corresponds to a degenerate hyperelliptic curve.

The associated second order supersymmetric Schrödinger system describes two reflectionless Pöschl-Teller systems with $m$ and $(m-1)$ bound states in their spectra. At $m=1$ one of the two Schrödinger subsystems is a non-relativistic free particle [7].

\section{B. Broken $\mathcal{N}=2$ supersymmetry and tri-supersymmetry in the light of the $\mathrm{nS}_{+}$hierarchy}

Now we consider the BdG system (3.5) defined by real $\Delta$ satisfying the $\mathrm{AKNS}_{N}$ equation with $N=2 m$. As we noted above, in this case the associated extended Schrödinger system (3.8) is characterized by the broken $\mathcal{N}=2$ supersymmetry. Our discussion will be restricted by three-gap BdG systems corresponding to the simplest case of $m=1$, for which the explicit solution of the AKNS equation can be found in both periodic and non-periodic cases. This will provide us, particularly, with a new structure of tri-supersymmetry, to which the $\mathcal{N}=2$ supersymmetry of the associated one-gap Schrödinger system is extended. One of its peculiar features is that in periodic and non-periodic cases the super-partner potentials are given by the same function shifted for an arbitrary distance $b$.

In the examples we discuss below, the nonlinear supersymmetric structure of the BdG system is based on the existence of the non-trivial local integral $S_{3}$ and a trivial nonlocal integral $\Gamma=\mathcal{R} \sigma_{2}$ playing the role of the $\mathbb{Z}_{2}$-grading operator. The first integral is a consequence of the $\mathrm{AKNS}_{2}$ equation satisfied by $\Delta$, while the second means that it is an even function, $\Delta(-x)=\Delta(x)$. Applying a chiral rotation, the latter integral can be reduced to the form of the grading operator we used in previous examples, i. e. $\mathcal{R} \sigma_{3}$. We do not apply, however, such a rotation here but work with the BdG Hamiltonian having the fixed off-diagonal form (3.5). 
From the viewpoint of the associated extended Schrödinger system, the non-trivial diagonal $2 \times 2$ matrix integral

$$
\mathcal{Z}=S_{3}
$$

which is the third order differential operator, is related with the stationary KdV hierarchy given by the Lax pair $(\mathcal{H}, \mathcal{Z}),[\mathcal{H}, \mathcal{Z}]=0$, where $\mathcal{H}=\mathcal{X}^{2}, \mathcal{X}=H_{\mathbb{R}}$. At the same time the next after $S_{3}$ trivial integral $S_{4}=S_{3} H_{\mathbb{R}}=H_{\mathbb{R}} S_{3}$ for the BdG system is also a trivial integral for the Schrödinger system, $S_{4}=\mathcal{Z} \mathcal{X}=\mathcal{X} \mathcal{Z}$, cf. (3.11) and (3.12).

Let us construct the matrix second order differential operator $S_{2}$ according to (2.2). A direct check shows that, as it should be expected, this operator is not an integral of motion for the BdG system,

$$
\left[H_{\mathbb{R}}, S_{2}\right]=i \epsilon(b) \sigma_{3} \quad,
$$

where $\epsilon(b)$ is some function of the displacement parameter, see Eqs. (3.53) and (3.66) below. But since $\mathcal{X}=$ $H_{\mathbb{R}}$ anticommutes with $\sigma_{3}$ (remember that this operator is a usual linear supercharge for the associated $\mathcal{N}=2$ superextended Schrödinger system $\mathcal{H}$, for which $\sigma_{3}$ is the grading operator), we get the relations

$$
\begin{gathered}
{[\mathcal{H}, \mathcal{X}]=[\mathcal{H}, \mathcal{Y}]=[\mathcal{H}, \mathcal{Z}]=0} \\
{[\mathcal{Z}, \mathcal{X}]=[\mathcal{Z}, \mathcal{Y}]=0} \\
{[\mathcal{X}, \mathcal{Y}]=i \epsilon(b) \sigma_{3}}
\end{gathered}
$$

where $\mathcal{Y}=S_{2}$. Therefore, as in the case of the exact $\mathcal{N}=2$ supersymmetry, the three operators $\mathcal{X}, \mathcal{Y}$ and $\mathcal{Z}$ are nontrivial integrals of motion for the associated Schrödinger system. With respect to the trivial integral $\sigma_{3}$ identified as the $\mathbb{Z}_{2}$-grading operator $\Gamma$, integrals $\mathcal{X}$ and $\mathcal{Y}$ are fermionic supercharges of the tri-supersymmetric structure, while $\mathcal{Z}$ is its bosonic central charge. Unlike the case of the exact $\mathcal{N}=2$ supersymmetry discussed above, here the fermionic supercharges do not commute, see (3.39). Nevertheless, in both cases they satisfy a relation

$$
\{\mathcal{X}, \mathcal{Y}\}=2 \mathcal{Z}
$$

Since the BdG Hamiltonian $H_{\mathbb{R}}$ anticommutes with $\sigma_{3}$ and $\mathcal{R} \sigma_{1}$, these operators alongside with $\mathcal{R} \sigma_{2}$ are the trivial integrals of motion for the associated Schrödinger system $\mathcal{H}$. Therefore, besides the usual identification $\Gamma=\sigma_{3}$, nonlocal operators $\mathcal{R} \sigma_{1}$ and $\mathcal{R} \sigma_{2}$ can also be chosen as $\mathbb{Z}_{2}$-grading operators for the associated Schrödinger system. Under the choice $\Gamma=\mathcal{R} \sigma_{1}$, the operators $\mathcal{X}$ and $\mathcal{Z}$ are identified as fermionic supercharges, while $\mathcal{Y}$ plays the role of the bosonic integral for the system $\mathcal{H}$. For alternative choice $\Gamma=\mathcal{R} \sigma_{2}, \mathcal{Z}$ is again a fermionic supercharge, while the (boson-fermion) nature of the operators $\mathcal{X}$ and $\mathcal{Y}$ is interchanged. For these two choices of the grading operator the commutator (3.39) is a part of a complete list of (anti)-commutation relations of the tri-supersymmetry of the Schrödinger system.

Let us stress that the essential difference of the described tri-supersymmetric structure of the Schrödinger system $\mathcal{H}$ in comparison with the tri-supersymmetry from Section IIA is that here the three trivial unitary involutive integrals of motion $\mathcal{R} \sigma_{1}, \mathcal{R} \sigma_{2}$ and $\sigma_{3}$ anticommute mutually and satisfy between themselves the same set of algebraic relations as the three Pauli sigma matrices. At the abstract level, they generate non-abelian quaternion group of order eight in contrast with the Klein four-group generated by trivial integrals of the tri-supersymmetric structure of the extended Schrödinger systems associated with the family of the finite-gap BdG systems discussed above.

\section{Real periodic solution of the second $n S_{+}$equation}

Let us consider the periodic function

$$
\begin{aligned}
\Delta(x) & =\frac{\operatorname{cn} b \operatorname{dn} b}{\operatorname{sn} b}+\nu \operatorname{sn} b \operatorname{sn}(x-b / 2) \operatorname{sn}(x+b / 2) \\
& =Z(b ; \nu)+\frac{\operatorname{cn} b \operatorname{dn} b}{\operatorname{sn} b}+Z(x-b / 2 ; \nu)-Z(x+b / 2 ; \nu),
\end{aligned}
$$

where $Z(x ; \nu)$ is the Jacobi Zeta function, and the real elliptic parameter is chosen, $0<\nu<1$. The real parameter $b$ lies in the range $0 \leq b \leq \mathbf{K}(\nu)$, where $\mathbf{K}(\nu)$ is the elliptic half-period. The function (3.41) is a solution of the third-order $\mathrm{AKNS}_{2}$ equation (to be more specific, in the terminology of [31], the second $\mathrm{nS}_{+}$equation):

$$
\Delta^{\prime \prime \prime}-6 \Delta^{2} \Delta^{\prime}-2\left(1+\nu-3 \mathrm{~ns}^{2} b\right) \Delta^{\prime}=0 .
$$


The Hamiltonian (3.5) takes the form

$$
H_{\mathbb{R}}=i\left(\begin{array}{cc}
0 & \frac{d}{d x}+\frac{\operatorname{cn} b \operatorname{dn} b}{\operatorname{sn} b}+\nu \operatorname{sn} b \operatorname{sn}(x-b / 2) \operatorname{sn}(x+b / 2) \\
\frac{d}{d x}-\left(\frac{\operatorname{cn} b \operatorname{dn} b}{\operatorname{sn} b}+\nu \operatorname{sn} b \operatorname{sn}(x-b / 2) \operatorname{sn}(x+b / 2)\right) & 0
\end{array}\right),
$$

and we can check that it has a trivial integral of motion $\Gamma=\mathcal{R} \sigma_{2},\left[\mathcal{R} \sigma_{2}, H_{\mathbb{R}}\right]=0$, where $\mathcal{R}$ means reflection. The square of (3.43) produces a self-isospectral Schrödinger operator with potentials $V^{ \pm}=\Delta^{2} \pm \Delta^{\prime}$ as in (3.6), satisfying the translation property $V^{-}(x)=V^{+}(x-b)$ :

$$
H_{R}^{2}=\left(\begin{array}{cc}
-\frac{d^{2}}{d x^{2}}+\mathrm{ns}^{2} b-1-\nu+2 \nu \mathrm{sn}^{2}(x+b / 2) & 0 \\
0 & -\frac{d^{2}}{d x^{2}}+\mathrm{ns}^{2} b-1-\nu+2 \nu \mathrm{sn}^{2}(x-b / 2)
\end{array}\right) .
$$

The spectrum of this system [see Figure 2 is composed of four bands, arranged symmetrically with respect to the zero energy level, which does not belong to the physical spectrum.

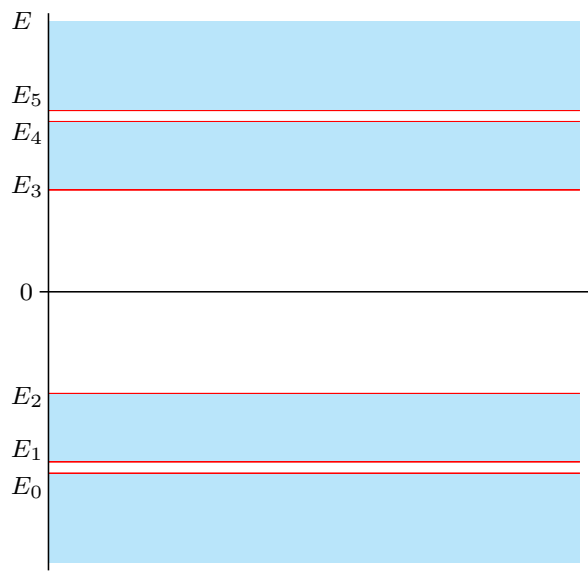

(a) $\nu=0.2, b=1$

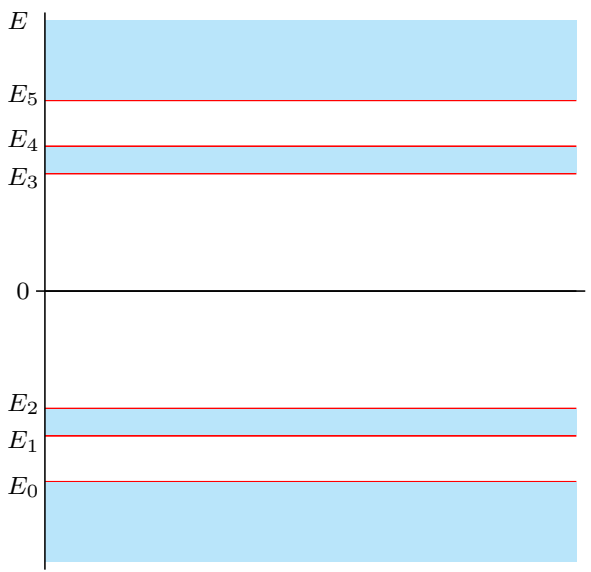

(c) $\nu=0.7, b=1$

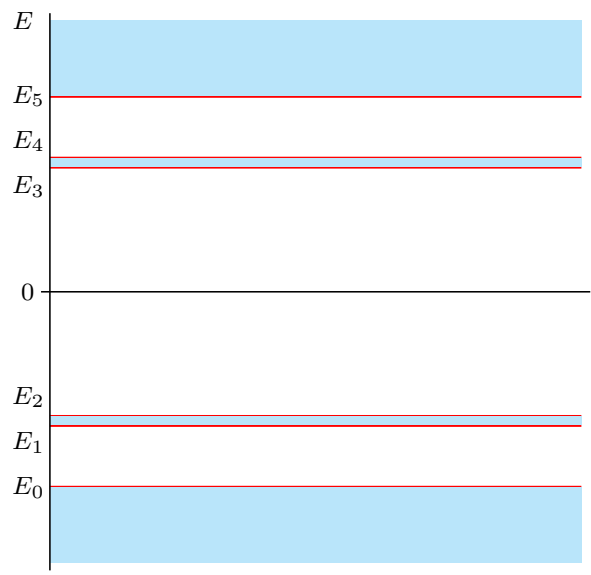

(b) $\nu=0.9, b=1$

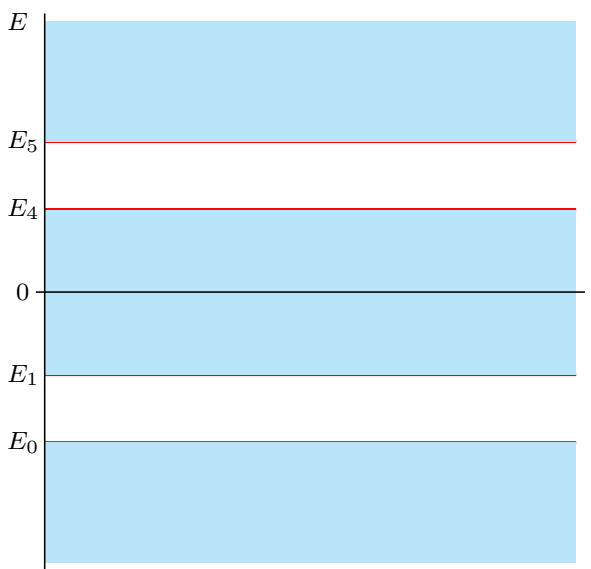

$(\mathrm{d}) \nu=0.7, b=\mathbf{K}(0.7)$

FIG. 2: Plots show the spectrum of 3.43) and its dependence on $\nu$ and $b$. The red lines correspond to the band edge energies. Figures (a) and (b) show the cases with different values of the modular parameter $\nu$ and the same value of $b$; in figures (c) and (d) the displacement constant $b$ varies, but $\nu$ is the same. The last plot (d) corresponds to the limit case when (3.41) is reduced to (3.14) with $m=1$.

The six band-edge states can be written easily in terms of the Jacobi elliptic functions

$$
\begin{gathered}
\psi_{0}=\left(\begin{array}{c}
i \operatorname{dn}(x+b / 2) \\
-\operatorname{dn}(x-b / 2)
\end{array}\right) \quad, \quad \psi_{1}=\left(\begin{array}{c}
i \operatorname{cn}(x+b / 2) \\
-\operatorname{cn}(x-b / 2)
\end{array}\right) \quad, \quad \psi_{2}=\left(\begin{array}{c}
i \operatorname{sn}(x+b / 2) \\
-\operatorname{sn}(x-b / 2)
\end{array}\right), \\
\psi_{3}=\sigma_{3} \psi_{2} \quad, \quad \psi_{4}=\sigma_{3} \psi_{1} \quad, \quad \psi_{5}=\sigma_{3} \psi_{0} \quad, \quad E_{2}=-E_{3}=-\frac{\operatorname{cn} b}{\operatorname{sn} b} .
\end{gathered}
$$


From the real $\mathrm{nS}_{+}$hierarchy we find a non-trivial integral of motion $S_{3}$ which annihilates all the band edge states,

$$
S_{3}=i\left(\begin{array}{rc}
S_{3}^{+} & 0 \\
0 & S_{3}^{-}
\end{array}\right) \quad, \quad\left[H_{\mathbb{R}}, S_{3}\right]=0
$$

where

$$
\begin{aligned}
& S_{3}^{+}(x)=\frac{d^{3}}{d x^{3}}+\left(1+\nu-3 \nu \operatorname{sn}^{2}(x+b / 2)\right) \frac{d}{d x}+3 i \nu \operatorname{dn}(x+b / 2) \operatorname{cn}(x+b / 2) \operatorname{sn}(x+b / 2) \\
& S_{3}^{-}(x)=S_{3}^{+}(x-b)
\end{aligned}
$$

The integral of motion $S_{3}$ anticommutes with $\mathcal{R} \sigma_{2}$, and we can identify the $\mathcal{N}=2$ nonlinear supersymmetry. It is generated by the supercharges

$$
\begin{gathered}
\mathcal{Q}_{1}=S_{3}, \quad \mathcal{Q}_{2}=i \mathcal{R} \sigma_{2} S_{3}, \\
{\left[H_{\mathbb{R}}, \mathcal{Q}_{a}\right]=0 \quad, \quad\left\{\mathcal{Q}_{a}, \mathcal{Q}_{b}\right\}=2 \delta_{a b} P_{6}\left(H_{\mathbb{R}}\right) \quad, \quad a, b=1,2,}
\end{gathered}
$$

where $P_{6}\left(H_{\mathbb{R}}\right)$ is a spectral polynomial

$$
P_{6}\left(H_{\mathbb{R}}\right)=\prod_{k=0}^{5}\left(H_{\mathbb{R}}-E_{k}\right)=\left(H_{\mathbb{R}}^{2}-E_{0}^{2}\right)\left(H_{\mathbb{R}}^{2}-E_{1}^{2}\right)\left(H_{\mathbb{R}}^{2}-E_{2}^{2}\right)
$$

Another operator from the hierarchy which commutes with $S_{3}$ but does not commute with $H_{\mathbb{R}}$ is

$$
S_{2}=i\left(\begin{array}{cc}
0 & S_{2}^{-} \\
S_{2}^{+} & 0
\end{array}\right) \quad, \quad\left[S_{2}, S_{3}\right]=0
$$

where

$$
S_{2}^{ \pm}=\frac{d^{2}}{d x^{2}} \mp \Delta \frac{d}{d x}-\frac{1}{2}\left(\Delta^{2} \mp \Delta^{\prime}-1-\nu+3 \mathrm{~ns}^{2} b\right)
$$

We can check that $S_{2}$ satisfies

$$
\left[H_{\mathbb{R}}, S_{2}\right]=2 i \sigma_{3} \frac{\operatorname{dn} b \operatorname{cn} b}{\operatorname{sn}^{3} b}
$$

Thus, this example shows how the nonlinear supersymmetry structure arises for the finite gap BdG system given by the periodic function (3.41). We note here that the Schrödinger system (3.44) was also discussed in [56] in the context of Darboux transformations.

\section{Real non-periodic solution of the second $n S_{+}$equation}

Now we consider the non-periodic limit of (3.41) when the elliptic parameter $\nu \rightarrow 1$,

$$
\Delta(x)=\operatorname{coth} b+\tanh (x-b / 2)-\tanh (x+b / 2)
$$

The main properties of the supersymmetric structure remain invariant in this limit. The gap function (3.54) is also a solution of the second $\mathrm{nS}_{+}$equation

$$
\Delta^{\prime \prime \prime}-6 \Delta^{2} \Delta^{\prime}+2\left(1+\frac{3}{\sinh ^{2} b}\right) \Delta^{\prime}=0 \quad .
$$

The Hamiltonian (3.5) takes here a form

$$
H_{\mathbb{R}}=i\left(\begin{array}{cc}
0 & \frac{d}{d x}+\operatorname{coth} b+\tanh (x-b / 2)-\tanh (x+b / 2) \\
\frac{d}{d x}-(\operatorname{coth} b+\tanh (x-b / 2)-\tanh (x+b / 2)) & 0
\end{array}\right.
$$


The square of $H_{\mathbb{R}}$ produces two self-isospectral reflectionless Schrödinger Hamiltonians,

$$
\mathcal{H}=H_{\mathbb{R}}^{2}=\left(\begin{array}{cc}
-\frac{d^{2}}{d x^{2}}-\frac{2}{\cosh ^{2}(x+b / 2)}+\operatorname{coth}^{2} b & 0 \\
0 & -\frac{d^{2}}{d x^{2}}-\frac{2}{\cosh ^{2}(x-b / 2)}+\operatorname{coth}^{2} b
\end{array}\right) .
$$

The spectrum of (3.56) is symmetric with respect to the zero level of energy. It is composed by two bound states which come from the shrink of the bands $\left[E_{1}, E_{2}\right] \rightarrow \mathcal{E}_{1}=-\frac{1}{\sinh b}$, and $\left[E_{3}, E_{4}\right] \rightarrow \mathcal{E}_{2}=\frac{1}{\sinh b}$,

$$
\psi_{1}=\left(\begin{array}{c}
i \operatorname{sech}(x+b / 2) \\
-\operatorname{sech}(x+b / 2)
\end{array}\right) \quad, \quad \psi_{2}=\sigma_{3} \psi_{1} \quad, \quad H_{\mathbb{R}} \psi_{i}=\mathcal{E}_{i} \psi_{i} \quad, \quad i=1,2,
$$

and by two continuous bands $\left(-\infty, \mathcal{E}_{0}\right]$ and $\left[\mathcal{E}_{4}, \infty\right)$. The energies in the continuous bands are doubly degenerate, except the singlet edge values, and are described by the eigenfunctions

$$
\begin{gathered}
\psi_{\uparrow}^{ \pm}=\left(\begin{array}{c}
\frac{ \pm k+i \operatorname{coth} b}{\mathcal{E}_{\uparrow ; k}} \mathcal{D}_{-1}^{+} e^{ \pm i k x} \\
\mathcal{D}_{-1}^{-} e^{ \pm i k x}
\end{array}\right), \quad H_{\mathbb{R}} \psi_{\uparrow}^{ \pm}=\mathcal{E}_{\uparrow ; k} \psi_{\uparrow}^{ \pm}, \quad \mathcal{E}_{\uparrow ; k}=\frac{\sqrt{\left(1+k^{2}\right) \sinh ^{2} b+1}}{\sinh b}, \\
\psi_{\downarrow}^{ \pm}=\sigma_{3} \psi_{\uparrow}^{ \pm}, \quad H_{\mathbb{R}} \psi_{\downarrow}^{ \pm}=\mathcal{E}_{\downarrow ; k} \psi_{\downarrow}^{ \pm}, \quad \mathcal{E}_{\downarrow ; k}=-\mathcal{E}_{\uparrow ; k} \quad,
\end{gathered}
$$

where we introduce the notation

$$
\mathcal{D}_{\lambda}^{ \pm}=\frac{d}{d x}+\lambda \tanh (x \pm b / 2)
$$

The singlet edge energies $\mathcal{E}_{\uparrow ; 0}=\mathcal{E}_{4}$ and $\mathcal{E}_{\downarrow ; 0}=\mathcal{E}_{0}$ correspond to zero value of the non-negative continuous parameter $k$.

The integral of motion $S_{3}$ of the corresponding Lax pair $\left(H_{\mathbb{R}}, S_{3}\right)$, which annihilates all the singlet states, can be obtained by taking limit $\nu \rightarrow 1$ in (3.46). It has the following structure:

$$
S_{3}=i\left(\begin{array}{cc}
\mathcal{D}_{-1}^{+} \mathcal{D}_{0}^{+} \mathcal{D}_{1}^{+} & 0 \\
0 & \mathcal{D}_{-1}^{-} \mathcal{D}_{0}^{-} \mathcal{D}_{1}^{-}
\end{array}\right)
$$

As in the periodic case we have

$$
\left[H_{\mathbb{R}}, \Gamma\right]=0 \quad, \quad\left\{S_{3}, \Gamma\right\}=0 \quad, \quad \Gamma=\sigma_{2} \mathcal{R} \quad
$$

and the supersymmetry has the same non linear structure (3.48), (3.49) with the spectral polynomial changed for

$$
P_{6}\left(H_{\mathbb{R}}\right)=\left(H_{\mathbb{R}}^{2}-\mathcal{E}_{0}^{2}\right)\left(H_{\mathbb{R}}^{2}-\mathcal{E}_{1}^{2}\right)^{2}
$$

The components of the operator $S_{2}$ from (3.51) reduce in the infinite period limit to

$$
S_{2}^{ \pm}=\frac{d^{2}}{d x^{2}} \mp(\operatorname{coth} b+\tanh (x-b / 2)-\tanh (x+b / 2)) \frac{d}{d x}+\frac{1}{\sinh ^{2} b}+\operatorname{sech}^{2}(x \pm b / 2) \quad .
$$

This operator obeys the relations

$$
\begin{gathered}
{\left[S_{2}, H_{\mathbb{R}}\right]=2 i \frac{\cosh b}{\sinh ^{3} b} \sigma_{3}} \\
{\left[S_{2}, S_{3}\right]=0}
\end{gathered}
$$

\section{COMPLEX CASE}

Here we describe a one-parametric generalization of the finite-gap systems from Section \A given by a complex $\Delta$. In such a case an energy reflection symmetry is partially broken, but the supersymmetric structure of the BdG system is preserved in a form to be coherent with the partial discrete symmetry breaking. A kind of duality underlying the supersymmetric structure is observed. 


\section{A. Complex non-periodic solution of the $(2 m-1)^{\text {th }} \mathbf{n S}_{+}$equation}

First, consider a simpler non-periodic limit case. We take the following complex generalization of the kink (3.25), the twisted kink [3, 57],

$$
\Delta(x ; \theta)=m \frac{\cosh (\sin (\theta / 2) x-i \theta / 2)}{\cosh (\sin (\theta / 2) x)} e^{i \theta / 2}
$$

where $m \in \mathbb{N}$, and $\theta$ is a real parameter. The function $\Delta(x)$ in 4.1 satisfies the complex $(2 m-1)^{\text {th }} \mathrm{nS}+$ equation. It obeys relations

$$
\Delta(x ; \theta+2 \pi)=\Delta(-x ; \theta) \quad, \quad \Delta(x, \theta+4 \pi)=\Delta(x ; \theta)
$$

and reduces to the real kink case (3.25) at $\theta=\pi$. It takes constant values $+m$ and $-m$ at $\theta=0$ and $\theta=2 \pi$ respectively, when the $\mathrm{BdG}$ system reduces to a free $(1+1) \mathrm{D}$ Dirac particle of mass $m$.

In correspondence with (4.2), the BdG Hamiltonian (1.24) anticommutes with the following two mutually commuting operators

$$
\mathcal{G}_{1}=-U\left(\frac{\theta}{2}\right) \gamma^{0} T_{2 \pi} U^{-1}\left(\frac{\theta}{2}\right) \quad, \quad \mathcal{G}_{2}=U\left(\frac{\theta}{2}\right) \mathcal{R} T_{2 \pi} U^{-1}\left(\frac{\theta}{2}\right)
$$

That is, $\left\{H, \mathcal{G}_{i}\right\}=0, i=1,2$, where $U$ is the operator of the chiral rotation defined in 3.1 , and $T_{2 \pi}$ is an operator of the half-period translation of $\theta, T_{2 \pi}: \theta \rightarrow \theta+2 \pi$. Notice that the treatment of the operators (4.3) in complex case $\theta \neq \pi$ implies the inclusion into consideration of a "dual" BdG system with shifted in $2 \pi$ value of the parameter $\theta$. Because of the presence of the operator $T_{2 \pi}$ in (4.3), the reflection energy symmetry of the real case, as we shall see below, is partially broken here. On the other hand, the half-period translation $T_{2 \pi}$ disappears in the composition of operators (4.3),

$$
\Gamma=\mathcal{R} U\left(\frac{\theta}{2}\right) \gamma^{5} U^{-1}\left(\frac{\theta}{2}\right)
$$

which is a nonlocal integral of motion of the system, $[\Gamma, H]=0$.

We present the BdG Hamitonian (1.24) in a more suitable form applying a unitary transformation given by the matrix

$$
\mathcal{U}_{\theta}=\frac{i}{\sqrt{2}}\left(\begin{array}{cc}
e^{-i \frac{\theta}{4}} & -e^{i \frac{\theta}{4}} \\
-e^{-i \frac{\theta}{4}} & -e^{i \frac{\theta}{4}}
\end{array}\right)
$$

which reduces to (3.3) when $\theta=\pi$. Equivalently, this corresponds to a choice of the following $\theta$-dependent representation for $\gamma$-matrices,

$$
\gamma^{0}=\left(\begin{array}{cc}
-i \sin \frac{\theta}{2} & \cos \frac{\theta}{2} \\
-\cos \frac{\theta}{2} & i \sin \frac{\theta}{2}
\end{array}\right) \quad, \quad \gamma^{1}=\left(\begin{array}{cc}
-\cos \frac{\theta}{2} & i \sin \frac{\theta}{2} \\
-i \sin \frac{\theta}{2} & \cos \frac{\theta}{2}
\end{array}\right) \quad, \quad \gamma^{5}=-\sigma_{1} \quad .
$$

In this representation the $\mathrm{BdG}$ Hamiltonian (1.24) acquires the form

$$
H_{\mathbb{C}}=i\left(\begin{array}{cc}
i m \cos \frac{\theta}{2} & \frac{d}{d x}+m \sin \frac{\theta}{2} \tanh \left(\sin \frac{\theta}{2} x\right) \\
\frac{d}{d x}-m \sin \frac{\theta}{2} \tanh \left(\sin \frac{\theta}{2} x\right) & -i m \cos \frac{\theta}{2}
\end{array}\right)=\left(\begin{array}{cc}
-m \cos \frac{\theta}{2} & i \hat{\mathcal{D}}_{m} \\
i \hat{\mathcal{D}}_{-m} & m \cos \frac{\theta}{2}
\end{array}\right)
$$

In (4.7) we introduced the same type of operators as in the previous section,

$$
\hat{\mathcal{D}}_{\lambda}=\frac{d}{d x}+\lambda \sin \frac{\theta}{2} \tanh \left(\sin \frac{\theta}{2} x\right),
$$

which satisfy the relation

$$
\hat{\mathcal{D}}_{\lambda}(x)=\sin \frac{\theta}{2} \mathcal{D}_{\lambda}\left(\sin \frac{\theta}{2} x\right)
$$

and reduce to $\mathcal{D}_{\lambda}$, defined in (3.26), when $\theta=\pi$. The operators defined in (4.3) take in representation (4.6) the form

$$
\mathcal{G}_{1}=\sigma_{3} T_{2 \pi} \quad, \quad \mathcal{G}_{2}=\mathcal{R} T_{2 \pi},
$$

and the nonlocal integral (4.4) reduces to (3.16), $\Gamma=\mathcal{R} \sigma_{3}$. 
Hamiltonian (4.7) satisfies the relation $T_{2 \pi} H_{\mathbb{C}}=\left(H_{\mathbb{C}}+2 m \cos \frac{\theta}{2} \sigma_{3}\right) T_{2 \pi}$, that transforms into $\left[T_{2 \pi}, H_{\mathbb{C}}\right]=0$ in the case $\theta=\pi$. Its eigenvalues depend on $\theta$ and satisfy the relation $\mathcal{E}(\theta+4 \pi)=\mathcal{E}(\theta)$ in correspondence with (4.2). Since $H_{\mathbb{C}}$ anticommutes with operators (4.10), we find particularly that if a state $\psi_{\mathcal{E}(\theta)}(x)$ is an eigenstate of (4.7) of eigenvalue $\mathcal{E}(\theta)$, then a state $\mathcal{R} T_{2 \pi} \psi_{\mathcal{E}(\theta)}(x)$ is an eigenstate of eigenvalue $-\mathcal{E}(\theta+2 \pi)$,

$$
H_{\mathbb{C}} \psi_{\mathcal{E}(\theta)}(x)=\mathcal{E}(\theta) \psi_{\mathcal{E}(\theta)}(x) \quad \Rightarrow \quad H_{\mathbb{C}} \mathcal{R} T_{2 \pi} \psi_{\mathcal{E}(\theta)}(x)=-\mathcal{E}(\theta+2 \pi) \mathcal{R} T_{2 \pi} \psi_{\mathcal{E}(\theta)}(x) \quad .
$$

Explicit form of the eigenstates and eigenvalues of the BdG Hamiltonian (4.7), discussed in Appendix, shows that for $\theta \neq 0(\bmod \pi)$, if $\mathcal{E}(\theta)$ is an energy of a physical state $\psi_{\mathcal{E}(\theta)}(x)$ to be different from the middle bound state, then the state $\sigma_{3} T_{2 \pi} \psi_{\mathcal{E}(\theta)}(x)$ (or, $\mathcal{R} T_{2 \pi} \psi_{\mathcal{E}(\theta)}(x)$ ) possesses the energy $-\mathcal{E}(\theta)$. Together with (4.11), this means that all the energy levels except the energy of the middle bound state are periodic functions of $\theta$ with the period $2 \pi$.

In more detail, as in the real case, for $\theta \neq 0,2 \pi$ the spectrum of (4.7) contains $(2 m+1)$ singlet states, $(2 m-1)$ of which correspond to bound states. The middle bound state and its energy are

$$
\psi_{m, 0}(x ; \theta)=\left(\begin{array}{c}
0 \\
\operatorname{sech}^{m}\left(\sin \frac{\theta}{2} x\right)
\end{array}\right) \quad, \quad \mathcal{E}_{m}(\theta)=m \cos \frac{\theta}{2} \quad, \quad H_{\mathbb{C}} \psi_{m, 0}(x ; \theta)=\mathcal{E}_{m}(\theta) \psi_{m, 0}(x ; \theta)
$$

Note that the energy $\mathcal{E}_{m}(\theta)=m \cos \frac{\theta}{2} \neq 0$ for $\theta \neq \pi$, and that it varies monotonically from $+m$ to $-m$ when $\theta$ varies in the interval $(0,2 \pi)$. For boundary values $\theta=0,2 \pi$ the bound state $\psi_{m, 0}(x ; \theta)$ as well as all other bound states disappear, and the system has a spectrum of a free massive (1+1)D Dirac particle consisting of two symmetric scattering bands $(-\infty,-m]$ and $[m, \infty)$. Thefore, for $\theta \neq 0(\bmod \pi)$ the energy reflection symmetry of the real case is partially broken.

Relations (4.11) and (4.12) show that the middle bound states of the two BdG systems with parameter $\theta$ shifted in $2 \pi$ satisfy the relation $\mathcal{E}_{m}(\theta)=-\mathcal{E}_{m}(\theta+2 \pi)$. This equality together with (4.11) correspond to a kind of duality relation

$$
H_{\mathbb{C}}(x ; \theta)=-H_{\mathbb{C}}(-x ; \theta+2 \pi),
$$

which is reflected in the structure of the spectrum shown on Fig. 3, cf. [58].

Observe that

$$
H_{\mathbb{C}}^{2}=\left(\begin{array}{cc}
-\frac{d^{2}}{d x^{2}}-\frac{m(m-1) \sin ^{2} \frac{\theta}{2}}{\cosh ^{2}\left(\sin \frac{\theta}{2} x\right)}+m^{2} & 0 \\
0 & -\frac{d^{2}}{d x^{2}}-\frac{m(m+1) \sin ^{2} \frac{\theta}{2}}{\cosh ^{2}\left(\sin \frac{\theta}{2} x\right)}+m^{2}
\end{array}\right)
$$

is diagonal, with Schrödinger-type Hamiltonians of Pöschl-Teller form on the diagonal. In the general complex case of the BdG Hamiltonian, this local diagonalization of $H^{2}$ is not possible.

The non-trivial integral of motion which comes from the Lax pair relation of the $n \mathrm{~S}_{+}$hierarchy can be presented in the form

$$
\begin{aligned}
S_{2 m} & =\left(\begin{array}{cc}
2 m i(-1)^{m} \cos \frac{\theta}{2} \hat{\mathcal{D}}_{-m+1}^{2 m-1} & \left(\hat{\mathcal{D}}_{-m}^{2 m}\right)^{\dagger} \\
\hat{\mathcal{D}}_{-m}^{2 m} & 0
\end{array}\right) \\
& =\left(\begin{array}{cc}
2 m i(-1)^{m} \cos \frac{\theta}{2} \hat{\mathcal{D}}_{-m+1} \hat{\mathcal{D}}_{-m+2} \ldots \hat{\mathcal{D}}_{m-1} & \hat{\mathcal{D}}_{-m+1} \hat{\mathcal{D}}_{-m+2} \ldots \hat{\mathcal{D}}_{m-1} \hat{\mathcal{D}}_{m} \\
\hat{\mathcal{D}}_{-m} \hat{\mathcal{D}}_{-m+1} \ldots \hat{\mathcal{D}}_{m-2} \hat{\mathcal{D}}_{m-1} & 0
\end{array}\right) .
\end{aligned}
$$

Since nonlocal operator $\mathcal{R} \sigma_{3}$ commutes with the Hamiltonian (4.7) and anticommutes with $S_{2 m}$,

$$
\left[\mathcal{R} \sigma_{3}, H_{\mathbb{C}}\right]=0 \quad, \quad\left\{\mathcal{R} \sigma_{3}, S_{2 m}\right\}=0
$$

we identify the operators

$$
\mathcal{Q}_{1}=S_{2 m} \quad, \quad \mathcal{Q}_{2}=i \mathcal{R} \sigma_{3} \mathcal{Q}_{1}
$$

as the supercharges. They generate the $\mathcal{N}=2$ polynomial superalgebra of the form (3.21),

$$
\left[\mathcal{Q}_{a}, H_{\mathbb{C}}\right]=0 \quad, \quad\left\{\mathcal{Q}_{a}, \mathcal{Q}_{b}\right\}=2 \delta_{a b} P_{4 m}\left(H_{\mathbb{C}}\right)
$$

in which the polynomial (3.34) becomes

$$
P_{4 m}\left(H_{\mathbb{C}}\right)=\left(H_{\mathbb{C}}-\mathcal{E}_{0}\right)\left(H_{\mathbb{C}}-\mathcal{E}_{2 m}\right) \prod_{i=1}^{2 m-1}\left(H_{\mathbb{C}}-\mathcal{E}_{i}\right)^{2}
$$




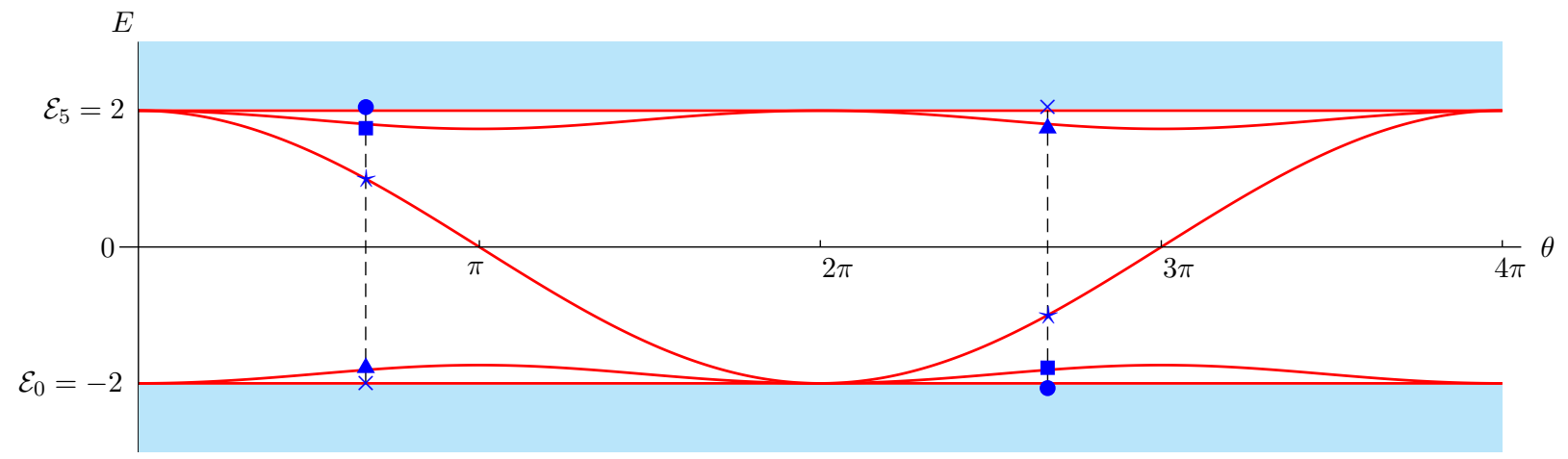

(a) $m=2$

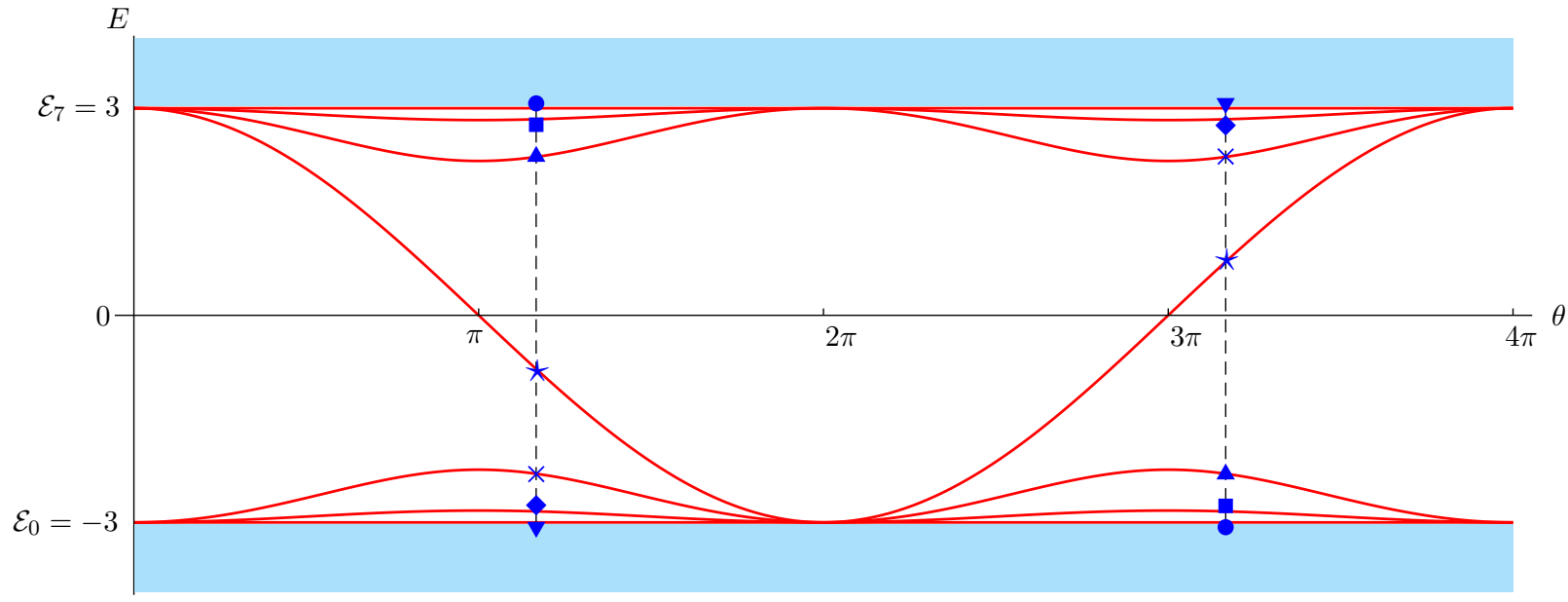

(b) $m=3$

FIG. 3: Spectrum of the BdG system (4.7) for $m=2$ and $m=3$. The red lines correspond to the singlet states energies; the middle red line reveals a partial breaking of the energy reflection symmetry. The pairs of the same symbols on the vertical lines indicate singlet energies for dual systems with shifted in $2 \pi$ parameter $\theta$.

Let us discuss now the nature of supersymmetry of the associated extended Schrödinger system described by the Hamiltonian (4.14). Though (4.14) is a square of the BdG Hamiltonian (4.7), the latter can not be treated as a usual supercharge of linear $\mathcal{N}=2$ supersymmetry with $\sigma_{3}$ identified as a $\mathbb{Z}_{2}$-grading operator. The reason is that though the quadratic diagonal operator (4.7) commutes with $\sigma_{3}$, the linear operator (4.7) does not anticommute with the diagonal Pauli matrix. In the rest of this section we present three distinct but related possible interpretations of the supersymmetry associated with the second order matrix diagonal Hamiltonian

$$
\mathcal{H}_{\mathbb{C}}=H_{\mathbb{C}}^{2}
$$

First, we note that the BdG Hamiltonian (4.7) can be presented as

$$
H_{\mathbb{C}}=H_{\mathbb{R}}^{\theta}-m \cos \frac{\theta}{2} \sigma_{3},
$$

where

$$
H_{\mathbb{R}}^{\theta}=\left(\begin{array}{cc}
0 & i \hat{\mathcal{D}}_{m} \\
i \hat{\mathcal{D}}_{-m} & 0
\end{array}\right)
$$

and $\hat{\mathcal{D}}_{m}$ is defined in (4.8). Operator (4.22) is the integral of motion for the system $\mathcal{H}_{\mathbb{C}}$. In correspondence with (4.9), it is the rescaled real BdG Hamiltonian $H_{\mathbb{R}}(x)$ defined in (3.26), $H_{\mathbb{R}}^{\theta}(x)=\sin \frac{\theta}{2} H_{\mathbb{R}}\left(\sin \frac{\theta}{2} x\right)$. Unlike the BdG Hamiltonian (4.21), it anticommutes with $\sigma_{3}$ and so, can be identified as one of two supercharges of the $\mathcal{N}=2$ linear supersymmetry, $\hat{\mathcal{Q}}_{1}=H_{\mathbb{R}}^{\theta}$. Defining the second supercharge in a usual way, $\hat{\mathcal{Q}}_{2}=i \sigma_{3} \hat{\mathcal{Q}}_{1}$, we arrive at the superalgebra

$$
\left\{\hat{\mathcal{Q}}_{a}, \hat{\mathcal{Q}}_{b}\right\}=2 \delta_{a b}\left(\mathcal{H}_{\mathbb{C}}-m^{2} \cos ^{2} \frac{\theta}{2}\right) \quad, \quad\left[\hat{\mathcal{Q}}_{a}, \mathcal{H}_{\mathbb{C}}\right]=0
$$


Since supercharges $\hat{\mathcal{Q}}_{a}$ are hermitian, from the first of these relations and relation (4.20) we find that the energies of the finite-gap BdG system (4.7) satisfy the inequality $\mathcal{E}^{2} \geq m^{2} \cos ^{2} \frac{\theta}{2}$. Since for $\theta \neq 0(\bmod 2 \pi)$ system (4.7) has an odd number of $2 m+1$ singlet states in its spectrum, the supersymmetric structure (4.23) of the associated Schrödinger system implies that the reflection energy symmetry is broken for $\theta \neq \pi(\bmod 2 \pi)$.

As in the real case $\theta=\pi$, relation (4.20) means that the BdG nontrivial integral (4.15) is also the integral of motion for the Schrödinger system $\mathcal{H}_{\mathbb{C}}$. Like the BdG Hamiltonian $H_{\mathbb{C}}$, integral $S_{2 m}$ does not have a definite $\mathbb{Z}_{2}$-parity with respect to the grading operator $\sigma_{3}$, but analogously to (4.21) it also can be decomposed into bosonic (diagonal) and fermionic (antidiagonal) operators

$$
S_{2 m}=\mathcal{F}^{\theta}+\mathcal{B}^{\theta}
$$

$\mathcal{F}^{\theta}$ is a rescaled nontrivial integral of a real case, $\mathcal{F}^{\theta}(x)=\left(\sin \frac{\theta}{2}\right)^{2 m} S_{\mathbb{R}}\left(\sin \frac{\theta}{2} x\right)$, with $S_{\mathbb{R}}(x)$ denoting here the operator (3.33). Bosonic operator is

$$
\mathcal{B}^{\theta}=2 m(-1)^{m} \cos \frac{\theta}{2} i \hat{\mathcal{D}}_{-m+1}^{2 m-1} \Pi_{+} \quad,
$$

where $\Pi_{+}=\frac{1}{2}\left(1+\sigma_{3}\right)$ is a projector. Both operators $\mathcal{F}^{\theta}$ and $\mathcal{B}^{\theta}$ are the integrals of motion for the Schrödinger system (4.20). Bosonic integral $\mathcal{B}^{\theta}$ can be related to a rescaled operator (3.11) with index $m$ changed for $m-1$. One can find a superlagebra generated by the fermionic set of integrals $\hat{\mathcal{Q}}_{a}, \mathcal{S}_{1}=\mathcal{F}^{\theta}, \mathcal{S}_{2}=i \sigma_{3} \mathcal{S}_{1}$, and by the bosonic integral $\mathcal{B}_{\theta}$ together with the Hamiltonian (4.20). It is a nonlinear extension of superalgebra (4.23), whose explicit form we do not display here.

Instead of hermitian integrals $\hat{\mathcal{Q}}_{a}, a=1,2$, associated with decomposition (4.21), consider now a pair of hermitian conjugate integrals

$$
Q=\left(\begin{array}{cc}
-\frac{m}{\sqrt{2}} \cos \frac{\theta}{2} & 0 \\
\hat{D}_{-m} & \frac{m}{\sqrt{2}} \cos \frac{\theta}{2}
\end{array}\right) \quad, \quad Q^{\dagger}=\left(\begin{array}{cc}
-\frac{m}{\sqrt{2}} \cos \frac{\theta}{2} & -\hat{D}_{m} \\
0 & \frac{m}{\sqrt{2}} \cos \frac{\theta}{2}
\end{array}\right)
$$

which are certain linear combinations of the integrals $\hat{\mathcal{Q}}_{1}, \hat{\mathcal{Q}}_{2}=i \sigma_{3} \hat{\mathcal{Q}}_{1}$ and $\sigma_{3}$. Together with the Schrödinger Hamiltonian $\mathcal{H}_{\mathbb{C}}$ they generate the centrally extended $\mathcal{N}=2$ superalgebra

$$
\begin{gathered}
\left\{Q, Q^{\dagger}\right\}=\mathcal{H}_{\mathbb{C}}, \quad\left[\mathcal{H}_{\mathbb{C}}, Q\right]=\left[\mathcal{H}_{\mathbb{C}}, Q^{\dagger}\right]=0, \\
\{Q, Q\}=\left\{Q^{\dagger}, Q^{\dagger}\right\}=\mathcal{C},
\end{gathered}
$$

in which the operator

$$
\mathcal{C}=m^{2} \cos ^{2} \frac{\theta}{2} I_{2},
$$

proportional to the $2 \times 2$ unit matrix $I_{2}$, plays the role of the central charge. Relation $\mathcal{H}_{\mathbb{C}} \geq \mathcal{C}$ is implied by superalgebra (4.27), (4.28) via relations $\left(H_{R}^{\theta}\right)^{2} \geq 0, H_{\mathbb{R}}^{\theta}=i\left(Q-Q^{\dagger}\right)$.

Central extension of $\mathcal{N}=2$ superalgebra of such a structure was discussed in [59] within the framework of supersymmetric quantum mechanics. Since mutually conjugate matrix integrals (4.26) do not anticommute with $\sigma_{3}$, they can not be identified as supercharges if $\sigma_{3}$ is treated as the $\mathbb{Z}_{2}$-grading operator $\Gamma$. In our case, however, we can identify one of the operators (4.10) as $\Gamma$. They both commute with $\mathcal{H}_{\mathbb{C}}$, and identify the Schrödinger Hamiltonian $\mathcal{H}_{\mathbb{C}}$ and the integral $\sigma_{3}$ as the bosonic operators, while integrals (4.26) are identified by any of them as the fermionic operators. Having in mind that $\left[\sigma_{3}, Q\right]=-2 i Q^{\dagger},\left[\sigma_{3}, Q^{\dagger}\right]=2 i Q$, the operator $\sigma_{3}$ can be treated then as a generator of $R$-symmetry.

Finally, let us discuss the tri-supersymmetric structure of the system (4.20). From the described properties of the finite-gap BdG system (4.7) it follows that the involutive operators (4.10) and their composition $\mathcal{R} \sigma_{3}$ are the trivial integrals of motion for the associated Schrödinger system [4.20), $\left[\mathcal{G}_{i}, \mathcal{H}_{\mathbb{C}}\right]=\left[\mathcal{R} \sigma_{3}, \mathcal{H}_{\mathbb{C}}\right]=0$. Let us denote $\mathcal{X}^{\theta}=H_{\mathbb{C}}$ and $\mathcal{Y}^{\theta}=S_{2 m}$, and introduce the operator

$$
\mathcal{Z}^{\theta}=\frac{1}{2}\left\{\mathcal{X}^{\theta}, \mathcal{Y}^{\theta}\right\}
$$

Operators $\mathcal{X}^{\theta}, \mathcal{Y}^{\theta}$ and $\mathcal{Z}^{\theta}$ are nontrivial integrals for the Schrödinger system 4.20), which at $\theta=\pi$ reduce to the integrals of the Schrödinger system corresponding to the real case. The choice of one of the three trivial integrals as 
the $\mathbb{Z}_{2}$-grading operator classifies the nontrivial integrals as bosonic and fermionic operators in correspondence with relations

$$
\begin{array}{rc}
{\left[\mathcal{X}^{\theta}, \mathcal{R} \sigma_{3}\right]=0 \quad, \quad\left\{\mathcal{Y}^{\theta}, \mathcal{R} \sigma_{3}\right\}=\left\{\mathcal{Z}^{\theta}, \mathcal{R} \sigma_{3}\right\}=0} \\
{\left[\mathcal{Y}^{\theta}, \mathcal{R} T_{2 \pi}\right]=0 \quad, \quad\left\{\mathcal{X}^{\theta}, \mathcal{R} T_{2 \pi}\right\}=\left\{\mathcal{Z}^{\theta}, \mathcal{R} T_{2 \pi}\right\}=0} \\
{\left[\mathcal{Z}^{\theta}, \sigma_{3} T_{2 \pi}\right]=0 \quad, \quad\left\{\mathcal{X}^{\theta}, \sigma_{3} T_{2 \pi}\right\}=\left\{\mathcal{Y}^{\theta}, \sigma_{3} T_{2 \pi}\right\}=0}
\end{array}
$$

As we noted above, for $\theta \neq \pi$ the treatment of the operators $\mathcal{R} T_{2 \pi}$ and $\sigma_{3} T_{2 \pi}$ requires a consideration of the pair of the corresponding dual systems.

\section{B. Complex periodic solution of the $(2 m-1)^{\text {th }} \mathrm{nS}_{+}$equation}

A periodic generalization of the function $\Delta(x)$ in (4.1) that also satisfies the $(2 m-1)^{\text {th }} \mathrm{nS}_{+}$equation and includes as special case the examples (real and complex) discussed above is [the $m=1$ case was studied in [3, 4] ]

$$
\Delta(x ; \theta)=-m A \frac{\sigma\left(A x+i \mathbf{K}^{\prime}-i \theta / 2\right)}{\sigma\left(A x+i \mathbf{K}^{\prime}\right) \sigma(i \theta / 2)} \exp \left[i A x(-i \zeta(i \theta / 2)+i \operatorname{ns}(i \theta / 2))+i \theta \eta_{3} / 2\right],
$$

where the parameter $A$ sets the scale, $A=A(\theta, \nu) \equiv-2 i \operatorname{sc}(i \theta / 4)$ nd $(i \theta / 4)$. The functions $\sigma=\sigma\left(x ; \omega_{1}, \omega_{2}\right)$ and $\zeta=\zeta\left(x ; \omega_{1}, \omega_{2}\right)$ are the Weierstrass sigma and zeta functions [53]. The real and imaginary half-periods are $\omega_{1}=\mathbf{K}(\nu)$, and $\omega_{3}=i \mathbf{K}^{\prime} \equiv i \mathbf{K}(1-\nu)$. The constant $\eta_{3} \equiv \zeta\left(i \mathbf{K}^{\prime}\right)$ is purely imaginary. The parameter $\theta$ plays the same role as in the previous non-periodic case, but now its period interval is $\left[0,8 \mathbf{K}^{\prime}(\nu)\right]$. Using the properties of elliptic functions one can check that for the specific values of the parameter $\theta=2 \mathbf{K}^{\prime}$ and $\theta=6 \mathbf{K}^{\prime}$, (4.34) reduces to the real periodic solution (3.14). In the infinite period limit $\nu \rightarrow 1$, the solution (4.34) reduces to the complex non-periodic case (4.1). Meanwhile for $\theta=0, \theta=4 \mathbf{K}^{\prime}$ or $\theta=8 \mathbf{K}^{\prime}$, the solution reduces to a free particle case given by $\Delta=b_{1} e^{i b_{2} x}$, where $b_{i}$, $i=1,2$ are constants, that produces a simple shift in the energy [3].

To analyze the properties of function (4.34), we decompose it in terms of the amplitude and phase; $\Delta=M e^{i \chi}$,

$$
\begin{aligned}
M(x) & =|\Delta|=m A \sqrt{\mathcal{P}\left(A x+i \mathbf{K}^{\prime}\right)-\mathcal{P}(i \theta / 2)}, \\
\chi(x) & =A(-i \zeta(i \theta / 2)+i \operatorname{ns}(i \theta / 2)) x+\frac{i}{2} \ln \left(\frac{\sigma\left(A x+i \mathbf{K}^{\prime}+i \theta / 2\right)}{\sigma\left(A x+i \mathbf{K}^{\prime}-i \theta / 2\right)}\right)+\frac{\eta_{3} \theta}{2}+\frac{\pi}{2},
\end{aligned}
$$

where $\mathcal{P}(x)$ is the elliptic Weierstrass function. Meanwhile $M$ is a function of period $T=2 \mathbf{K} / A$ in $x, \chi$ changes in $\varphi=2 \mathbf{K}\left(-i \zeta(i \theta / 2)+i \operatorname{ns}(i \theta / 2)-\frac{\eta \theta}{2 \mathbf{K}}\right)$ over the period $T$. As a result (4.34) changes as $\Delta(x+2 \mathbf{K} / A)=e^{i \varphi} \Delta(x)$, , i. e. (4.34) is a quasi-periodic in $x$ function. Hence, in correspondence with (3.1), the BdG Hamiltonian (1.1) satisfies the relation

$$
H(x+T)=e^{i \gamma_{5} \varphi / 2} H(x) e^{-i \gamma_{5} \varphi / 2}
$$

i. e. it is periodic up to a chiral transformation. This means that $H(x+T)$ and $H(x)$ systems are unitary equivalent, and the BdG Hamiltonian with quasi-periodic complex function (4.34) describes the finite-gap periodic system.

The spectrum of this system shown on Figure 4 has the properties similar to those we described in the non-periodic case. The continuum bands $\left(-\infty, E_{0}\right]$ and $\left[E_{4 m-1}, \infty\right)$, as well as any corresponding pair from $2 m-2$ valence bands which do not include the middle band are symmetric with respect to the zero energy level. As in the non-periodic case, the spectrum reveals also a symmetry associated with a duality relation, which, up to a unitary chiral transformation, has the form of (4.13) with $2 \pi$ changed here for the $\theta$-half-period equal to $4 \mathbf{K}^{\prime}(\nu)$ [72].

To reveal the $\mathcal{N}=2$ nonlinear supersymmetric structure and the partial breaking of the energy reflection symmetry in the finite-gap BdG system given by (4.34), we identify first the $\mathbb{Z}_{2}$-grading operator. To this aim we note that the imaginary part of (4.34) is an even function, while its real part is parity-odd. Applying the unitary transformation (4.5) with parameter $\theta=\pi$ to the BdG Hamiltonian (1.1) we obtain

$$
H_{\mathbb{C}}=\left(\begin{array}{cc}
\frac{i}{2}\left(\Delta-\Delta^{*}\right) & i \frac{d}{d x}+\frac{i}{2}\left(\Delta^{*}+\Delta\right) \\
i \frac{d}{d x}-\frac{i}{2}\left(\Delta^{*}+\Delta\right) & -\frac{i}{2}\left(\Delta-\Delta^{*}\right)
\end{array}\right)=\left(\begin{array}{cc}
-\operatorname{Im} \Delta & i\left(\frac{d}{d x}+\operatorname{Re} \Delta\right) \\
i\left(\frac{d}{d x}-\operatorname{Re} \Delta\right) & \operatorname{Im} \Delta
\end{array}\right)
$$




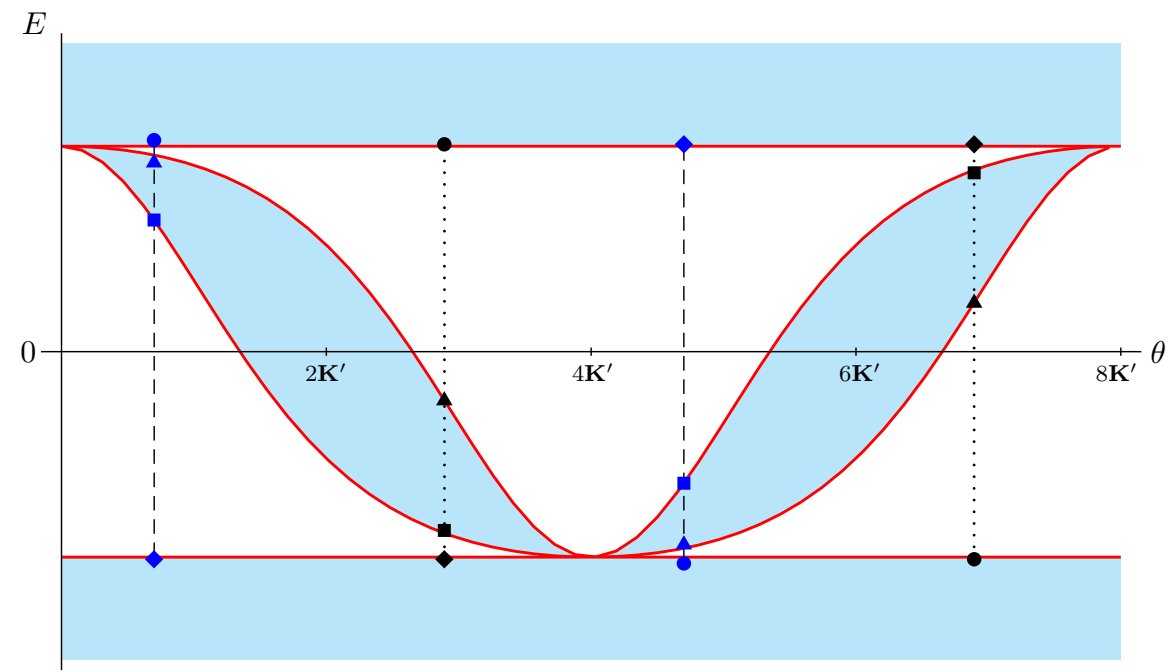

FIG. 4: Spectrum of the BdG system for the complex periodic solution (4.34) with $m=1$ in dependence on $\theta$. The pairs of the same symbols on the red lines corresponding to the singlet edge-states reflect a symmetry associated with the duality between the pairs of the systems with parameter $\theta$ shifted in the half-period $4 \mathbf{K}^{\prime}(\nu)$.

The diagonal and anti-diagonal parts of the transformed BdG Hamiltonian have opposite parities, and similarly to the antiperiodic case, (4.38) commutes with the nonlocal operator $\mathcal{R} \sigma_{3},\left[\mathcal{R} \sigma_{3}, H_{\mathbb{C}}\right]=0$. We can also find the analogs of the the operators (4.3) which factorize the integral $\mathcal{R} \sigma_{3}$ and anticommute with (4.38). These are

$$
\begin{gathered}
\mathcal{G}_{1}=\sigma_{3} T_{4 \mathbf{K}^{\prime}}, \quad \mathcal{G}_{2}=\mathcal{R} T_{4 \mathbf{K}^{\prime}}, \\
\left\{\mathcal{G}_{i}, H_{\mathbb{C}}\right\}=0 \quad, \quad i=1,2 .
\end{gathered}
$$

Here we introduced the half-period $\theta$-translation operator $T_{4 \mathbf{K}^{\prime}}: \theta \rightarrow \theta+4 \mathbf{K}^{\prime}$.

As in the previous non-periodic case, from relation (2.5) we can construct an operator $S_{2 m}$ which is a nontrivial integral of motion $\left[H_{\mathbb{C}}, S_{2 m}\right]=0$. In contrast with the previous cases, here it is not easy to find a closed general form for this operator, and we just refer to Eq. (2.5). Conserved quantity $S_{2 m}$ anti-commutes with integral $\mathcal{R} \sigma_{3}$, that allows us to identify the integrals $\mathcal{Q}_{1}=S_{2 m}$ and $\mathcal{Q}_{2}=i \mathcal{R} \sigma_{3} \mathcal{Q}_{1}$ as supercharges. They generate the nonlinear $\mathcal{N}=2$ supersymmetry,

$$
\left[\mathcal{Q}_{a}, H_{\mathbb{C}}\right]=0 \quad, \quad\left\{\mathcal{Q}_{a}, \mathcal{Q}_{b}\right\}=2 \delta_{a b} P_{4 m}\left(H_{\mathbb{C}}\right)
$$

where

$$
P_{4 m}\left(H_{\mathbb{C}}\right)=\prod_{i=1}^{4 m-1}\left(H_{\mathbb{C}}-E_{i}\right)
$$

is the spectral polynomial, and $E_{i}$ are the band-edge energies. In the limit $\nu \rightarrow 1$, (4.42) reduces to the polynomial (4.19) of the non-periodic case.

The square of the BdG Hamiltonian (4.38) takes here a non-diagonal form in contrast with the diagonal form of the associated Schrödinger Hamiltonian we had in the non-periodic case. Explicitly, we find that

$$
\mathcal{H}_{\mathbb{C}}=H_{\mathbb{C}}^{2}=\left(\begin{array}{cc}
-\frac{d^{2}}{d x^{2}}+\operatorname{Re}^{2} \Delta+(\operatorname{Re} \Delta)^{\prime}+\operatorname{Im}^{2} \Delta & i(\operatorname{Im} \Delta)^{\prime} \\
-i(\operatorname{Im} \Delta)^{\prime} & -\frac{d^{2}}{d x^{2}}+\operatorname{Re}^{2} \Delta-(\operatorname{Re} \Delta)^{\prime}+\operatorname{Im}^{2} \Delta
\end{array}\right)
$$

includes purely imaginary off-diagonal terms. The second order Hamiltonian (4.43) can be written in a more simple form if we define the matrix superpotential $\mathcal{W}$

$$
\mathcal{W}=\operatorname{Re} \Delta \sigma_{3}-\operatorname{Im} \Delta \sigma_{2} \quad, \quad \mathcal{H}_{\mathbb{C}}=-\frac{d^{2}}{d x^{2}}+\mathcal{W}^{2}+\mathcal{W}^{\prime}
$$


Though the second order matrix Hamiltonian includes the off-diagonal terms, which can be interpreted as a coupling between the partner subsystems, the tri-supersymmetric structure still presents in this case as well. With the identification of operators $\mathcal{X}^{\theta}=H_{\mathbb{C}}, \mathcal{Y}^{\theta}=S_{2 m}$ and $\mathcal{Z}^{\theta}=\frac{1}{2}\{\mathcal{X}, \mathcal{Y}\}$, as in the limit case (4.30) when $\nu \rightarrow 1$, we can treat them as nontrivial integrals of the second order system $\mathcal{H}_{\mathbb{C}}$. Their fermionic-bosonic nature and the concrete form of the corresponding superalgebra depend on which of the three trivial integrals

$$
\mathcal{R} \sigma_{3} \quad, \quad \mathcal{R} T_{4 \mathbf{K}^{\prime}} \quad, \quad \text { or } \quad \sigma_{3} T_{4 \mathbf{K}^{\prime}}
$$

is identified as the $\mathbb{Z}_{2}$-grading operator, cf. Eqs. (4.31)-4.33).

\section{CONCLUSIONS}

The Bogoliubov/de Gennes Hamiltonian (1.24) appears in a wide variety of contexts in theoretical and mathematical physics. Here we have shown first that the Ginzburg-Landau expansion of the associated grand canonical potential has a recursive structure that is precisely that of the AKNS integrable hierarchy. This explains why it is possible to solve the corresponding inhomogeneous gap equation by a simple ansatz for the Gorkov resolvent, as was done in [3]. We further have shown that this integrable AKNS hierarchy structure, and its finite gap solutions, provide the natural formalism for characterizing nonlinear quantum mechanical supersymmetry and tri-supersymmetry. We have illustrated these ideas with explicit solutions in terms of trigonometric and elliptic functions, for which the spectral and quantum mechanical supersymmetry properties can be computed in closed-form. Mathematically, there are open questions regarding the explicit form of the multi-dimensional theta function solutions to the $\mathrm{AKNS}_{N}$ hierarchy equations for $N \geq 2$, which are associated with hyperelliptic [rather than elliptic] curves, and Riemann surfaces of genus $g \geq 2$ [13, 31, 37, 60]. This is deeply related to the problem of the reduction of abelian integrals on Riemann surfaces [31, 37, 60, 61]. An interesting case that is not yet well understood in such explicit terms is the complex AKNS solution with an even number of gaps. Physically, interesting open problems are: (i) the question of the existence of self-consistent solutions $\Delta(x)$ to the inhomogeneous gap equation for the massive $\mathrm{NJL}_{2}$ model, for which approximate solutions are known [62], but no analytic solutions are known; (ii) the search for self-consistent crystalline solutions in higher dimensional models [46, 63, 64]. It would also be interesting to apply these integrability and quantum mechanical supersymmetry structures to the generalized Gross-Neveu and NJL models coming from string theories [65, 66, 67, 68].

\section{APPENDIX}

In this Appendix we present the solution of the eigenvalue problem for the non-periodic BdG Hamiltonian (4.7) in terms of solution of the same problem for the real case $\theta=\pi$ (3.26). These two problems can be related in a simple way because of a very special nature of the BdG Hamiltonian corresponding to the twisted kink (4.1): in general case, in contrast with (4.7), the square of (1.1) can not be transformed into a local diagonal form.

Let us denote (3.28) and (3.31) universally by $\psi_{+}$and $\psi_{-}$as solutions with positive and negative energy respectively. As we noted, for zero energy eigenvalue, Eqs. (3.28) and (3.31) give the same eigenstate (3.30). Due to the energy reflection symmetry of the real case, eigenfunctions of positive and negative energy sectors are related as $\psi_{-}=\sigma_{3} \psi_{+}$. We have

$$
H_{\mathbb{R}}^{\theta}(x) \tilde{\psi}_{ \pm}(x)= \pm \sin \frac{\theta}{2} \tilde{\mathcal{E}}_{+} \tilde{\psi}_{ \pm}(x) \quad, \quad \tilde{\psi}_{ \pm}(x)=\psi_{ \pm}\left(\sin \frac{\theta}{2} x\right)
$$

where $H_{\mathbb{R}}^{\theta}(x)=\sin \frac{\theta}{2} H_{\mathbb{R}}\left(\sin \frac{\theta}{2} x\right)$ corresponds to the notation introduced in (4.21), and by $\tilde{\mathcal{E}}_{+}$we denoted the eigenvalue of the state $\psi_{+}, H_{\mathbb{R}} \psi_{+}=\tilde{\mathcal{E}}_{+} \psi_{+}$. Using relation (4.21), we can rewrite (6.1) as

$$
\left(H_{\mathbb{C}}+m \cos \frac{\theta}{2} \sigma_{3}\right) \tilde{\psi}_{+}=\sin \frac{\theta}{2} \tilde{\mathcal{E}}_{+} \tilde{\psi}_{+} \quad, \quad\left(H_{\mathbb{C}}+m \cos \frac{\theta}{2} \sigma_{3}\right) \sigma_{3} \tilde{\psi}_{+}=-\sin \frac{\theta}{2} \tilde{\mathcal{E}}_{+} \sigma_{3} \tilde{\psi}_{+} .
$$

From here we find the eigenvalues and eigenstates of the Hamiltonian $H_{\mathbb{C}}$,

$$
\begin{gathered}
H_{\mathbb{C}} \psi_{ \pm}(x ; \theta)= \pm \mathcal{E}_{+}(\theta) \psi_{ \pm}(x ; \theta) \\
\mathcal{E}_{+}(\theta)=\sqrt{\sin ^{2} \frac{\theta}{2} \tilde{\mathcal{E}}_{+}^{2}+m^{2} \cos ^{2} \frac{\theta}{2}} \quad, \quad \psi_{ \pm}(x ; \theta)=\left(\alpha_{ \pm}(\theta)+\sigma_{3}\right) \tilde{\psi}_{+}(x),
\end{gathered}
$$


where

$$
\alpha_{+}(\theta)=\frac{-m \cos \frac{\theta}{2}}{\mathcal{E}_{+}(\theta)-\sin \frac{\theta}{2} \tilde{\mathcal{E}}_{+}} \quad, \quad \alpha_{-}(\theta)=\alpha_{+}(\theta+2 \pi) .
$$

Note that the middle bound state energy $\mathcal{E}_{m}(\theta)=m \cos \frac{\theta}{2}$, for which $\tilde{\mathcal{E}}_{+}=\mathcal{E}_{+}(\pi)=0$ in (6.4), is antiperiodic function of the parameter $\theta, \mathcal{E}_{m}(\theta+2 \pi)=-\mathcal{E}_{m}(\theta)$. For the rest of the spectrum, energy levels change periodically in $\theta$ with the period $2 \pi$, that is reflected coherently in Fig. 3 ,

\section{ACKNOWLEDGMENTS}

GD thanks the DOE for support under grant DE-FG02-92ER40716, and is particularly grateful to F. Gesztesy and V. Enolskii for their valuable comments. FC acknowledges the support from CONICYT, and thanks the Physics Department of the University of Connecticut for support and hospitality during an extended visit. MP acknowledges the support from FONDECYT under the grant 1095027.

[1] P. G. de Gennes, Superconductivity of Metals and Alloys, (Addison-Wesley, Redwood City, 1989).

[2] V. Schön and M. Thies, "2D model field theories at finite temperature and density," arXiv:hep-th/0008175, in Shifman, M. (ed.), At the frontier of particle physics, Vol. 3 (World Scientific); M. Thies, "From relativistic quantum fields to condensed matter and back again: Updating the Gross-Neveu phase diagram," J. Phys. A 39, 12707 (2006) arXiv:hep-th/0601049.

[3] G. Basar and G. V. Dunne, "Self-consistent crystalline condensate in chiral Gross-Neveu and Bogoliubov-de Gennes systems," Phys. Rev. Lett. 100, 200404 (2008), arXiv:0803.1501 [hep-th]; G. Basar and G. V. Dunne, "A Twisted Kink Crystal in the Chiral Gross-Neveu model," Phys. Rev. D 78, 065022 (2008), arXiv:0806.2659 [hep-th].

[4] G. Basar, G. V. Dunne and M. Thies, "Inhomogeneous Condensates in the Thermodynamics of the Chiral NJL 2 model," arXiv:0903.1868 [hep-th].

[5] D. J. Gross and A. Neveu, "Dynamical Symmetry Breaking In Asymptotically Free Field Theories," Phys. Rev. D 10, 3235 (1974).

[6] R. F. Dashen, B. Hasslacher and A. Neveu, "Semiclassical Bound States In An Asymptotically Free Theory," Phys. Rev. D 12, $2443(1975)$.

[7] A. Neveu and N. Papanicolaou, "Integrability Of The Classical Scalar And Symmetric Scalar-Pseudoscalar Contact Fermi Interactions In Two-Dimensions," Commun. Math. Phys. 58, 31 (1978).

[8] K. G. Klimenko, "Phase Structure Of Generalized Gross-Neveu Models," Z. Phys. C 37, 457 (1988).

[9] J. Feinberg and A. Zee, "Generalized supersymmetric quantum mechanics and reflectionless fermion bags in 1+1 dimensions," arXiv:hep-th/0109045, in Olshanetsky, M. (ed.) et al. Multiple facets of quantization and supersymmetry (World Scientific, 2002); J. Feinberg, "All about the static fermion bags in the Gross-Neveu model," Annals Phys. 309, 166 (2004) arXiv:hep-th/0305240.

[10] R. Jackiw and C. Rebbi, "Solitons With Fermion Number 1/2," Phys. Rev. D 13, 3398 (1976).

[11] A. J. Niemi and G. W. Semenoff, "Fermion Number Fractionization In Quantum Field Theory," Phys. Rept. 135, 99 (1986).

[12] R. Peierls, The Quantum Theory of Solids (Oxford, 1955).

[13] E. D. Belokolos et al, Algebro-geometric approach to nonlinear integrable equations, (Springer, Berlin, 1994).

[14] S. A. Brazovskii, S. A. Gordynin, and N. N. Kirova, "Exact solution of the Peierls model with an arbitrary number of electrons in the unit cell", Pis. Zh. Eksp. Teor. Fiz. 31, 486 (1980) [JETP Lett. 31456 (1980)]; "Excitons, polarons and bipolarons in conducting polymers", Pis. Zh. Eksp. Teor. Fiz. 33, 6 (1981) [JETP Lett. 33, 4 (1981)]; S. A. Brazovskii, N. N. Kirova and S.I. Matveenko, "Peierls effect in conducting polymers", Zh. Eksp. Teor. Fiz. 86, 743 (1984) [Sov. Phys. JETP 59, 434 (1984)].

[15] B. Horovitz, "Soliton Lattice in Polyacetylene, Spin-Peierls Systems, and Two-Dimensional Sine-Gordon Systems", Phys. Rev. Lett. 46, 742 (1981).

[16] D. K. Campbell and A. R. Bishop, "Soliton Excitations In Polyacetylene And Relativistic Field Theory Models," Nucl. Phys. B 200, 297 (1982).

[17] A. J. Heeger, S. Kivelson, J. R. Schrieffer and W. P. Su, "Solitons in conducting polymers," Rev. Mod. Phys. 60, 781 (1988).

[18] G. Eilenberger, "Transformation of Gorkov's Equation for Type II Superconductors into Transport Like Equations", Z. Phys. 214, 195 (1968).

[19] J. Bar-Sagi and C. G. Kuper, "Self-Consistent Pair Potential in an Inhomogeneous Superconductor", Phys. Rev. Lett. 28 1556 (1972).

[20] A. I. Buzdin and V. V. Tugushev, "Phase diagrams of electronic and superconducting transitions to soliton lattice states", Zh. Eksp. Teor. Fiz. 85, 735 (1983), [Sov. Phys. JETP 58, 428 (1983)]; A. I. Buzdin and S. V. Polonskii, "Nonuniform State in quasi-1D superconductors", Zh. Eksp. Teor. Fiz. 93, 747 (1987), [Sov. Phys. JETP 66, 422 (1988)]. 
[21] I. Kosztin, S. Kos, M. Stone and A. J. Leggett, "Free energy of an inhomogeneous superconductor: A wave-function approach", Phys. Rev. B 58, 9365 (1998); S. Kos and M. Stone, "Gradient expansion for the free energy of a clean superconductor", Phys. Rev. B 59, 9545 (1999).

[22] K. Machida and H. Nakanishi, "Superconductivity under a ferromagnetic molecular field", Phys. Rev. B 30, 122 (1984).

[23] J. Mertsching and H. J. Fischbeck, "The incommensurate Peierls phase of the quasi-dimensional Frohlich model with a nearly half-filled band", Phys. Stat. Sol. B 103, 783 (1981).

[24] Z. b. Su and B. Sakita, "Chiral symmetry and chiral anomaly in an incommensurate charge-density-wave system," Phys. Rev. Lett. 56, 780 (1986).

[25] S. Giorgini, L. P. Pitaevskii and S. Stringari, "Theory of ultracold Fermi gases", Rev. Mod. Phys. 80, 1215 (2008).

[26] A. A. Andrianov, M. V. Ioffe and V. P. Spiridonov, "Higher derivative supersymmetry and the Witten index," Phys. Lett. A 174, 273 (1993) arXiv:hep-th/9303005.

[27] S. M. Klishevich and M. S. Plyushchay, "Nonlinear supersymmetry, quantum anomaly and quasi-exactly solvable systems," Nucl. Phys. B 606, 583 (2001) arXiv:hep-th/0012023.

[28] L. P. Gorkov, Zh. ksp. Teor. Fiz. 34, 735 (1958) [Sov. Phys. JETP 7, 505 (1958)].

[29] L. A. Dickey, Soliton Equations and Hamiltonian Systems, (World Scientific, Singapore, 1991).

[30] A. Das, Integrable Models, (World Scientific, Singapore, 1989).

[31] F. Gesztesy and H. Holden, Soliton Equations and their Algebro-Geometric Solutions, (Cambridge University Press, 2003).

[32] J. Kapusta, Finite Temperature Field Theory, (Cambridge Univ. Press, 1989).

[33] D. Waxman, "The Fredholm Determinant For A Dirac Hamiltonian With A Topological Mass Term," Annals Phys. 241, 285 (1995).

[34] A. M. Perelomov, "Schrödinger equation spectrum and Korteweg-De Vries type invariants", Ann. de l'inst. H. Poincaré 24, 161 (1976); A. M. Perelomov and Y. Zeldovich, Quantum Mechanics: Selected Topics, (World Scientific, Singapore, 1998).

[35] M. J. Ablowitz, D. J. Kaup, A. C. Newell and H. Segur, "The Inverse scattering transform fourier analysis for nonlinear problems," Stud. Appl. Math. 53, 249 (1974).

[36] P. D. Lax, "Integrals Of Nonlinear Equations Of Evolution And Solitary Waves," Commun. Pure Appl. Math. 21, 467 (1968).

[37] F. Gesztesy and R. Weikard, "A characterization of all elliptic algebro-geometric solutions of the AKNS hierarchy", Acta Math. 181, 63 (1998); "Elliptic Algebro-geometric solutions of the KdV and AKNS hierarchies - an analytic approach", Bull. Am. Math. Soc. 35, 271 (1998).

[38] A. O. Smirnov, "The Dirac Operator with Elliptic Potential", Sbornik Math 186, 1213 (1995).

[39] M. S. Plyushchay, "Deformed Heisenberg algebra, fractional spin fields and supersymmetry without fermions," Annals Phys. 245, 339 (1996) arXiv:hep-th/9601116]; "Hidden nonlinear supersymmetries in pure parabosonic systems," Int. J. Mod. Phys. A 15, 3679 (2000) arXiv:hep-th/9903130.

[40] F. Correa and M. S. Plyushchay, "Hidden supersymmetry in quantum bosonic systems," Annals Phys. 322, 2493 (2007) arXiv:hep-th/0605104]; "Peculiarities of the hidden nonlinear supersymmetry of Poschl-Teller system in the light of Lamé equation," J. Phys. A 40, 14403 (2007) [arXiv:hep-th/0706.1114].

[41] F. Correa, L. M. Nieto and M. S. Plyushchay, "Hidden nonlinear supersymmetry of finite-gap Lame equation," Phys. Lett. B 644, 94 (2007) arXiv:hep-th/0608096.

[42] E. Witten, "Dynamical Breaking Of Supersymmetry," Nucl. Phys. B 188, 513 (1981).

[43] F. Cooper, A. Khare and U. Sukhatme, "Supersymmetry and quantum mechanics," Phys. Rept. 251, 267 (1995) arXiv:hep-th/9405029.

[44] A. K. Grant and J. L. Rosner, "Supersymmetric quantum mechanics and the Korteweg-de Vries hierarchy," J. Math. Phys. 35, 2142 (1994) arXiv:hep-th/9304139.

[45] V. B. Matveev and M. A. Salle, Darboux Transformations and Solitons, (Springer, Berlin, 1991).

[46] G. Dunne, H. Gies, K. Klingmuller and K. Langfeld, "Worldline Monte Carlo for fermion models at large N , $^{\circ}$ arXiv:0903.4421 [hep-th].

[47] G. V. Dunne and J. Feinberg, "Self-isospectral periodic potentials and supersymmetric quantum mechanics," Phys. Rev. D 57, 1271 (1998) arXiv:hep-th/9706012.

[48] J. L. Burchnall and T. W. Chaundy, "Commutative ordinary differential operators," Proc. Lond. Math. Soc. 21, 420 (1923).

[49] E. L. Ince, Ordinary differential equations, (Dover, 1956).

[50] F. Correa, L. M. Nieto and M. S. Plyushchay, "Hidden nonlinear su(2|2) superunitary symmetry of N=2 superextended 1D Dirac delta potential problem," Phys. Lett. B 659, 746 (2008) arXiv:0707.1393 [hep-th].

[51] F. Correa, V. Jakubský, L. M. Nieto and M. S. Plyushchay, "Self-isospectrality, special supersymmetry, and their effect on the band structure," Phys. Rev. Lett. 101, 030403 (2008), arXiv:0801.1671 [hep-th].

[52] F. Correa, V. Jakubský and M. S. Plyushchay, "Finite-gap systems, tri-supersymmetry and self-isospectrality," J. Phys. A 41, 485303 (2008) arXiv:0806.1614 [hep-th].

[53] D. F. Lawden, Elliptic Functions and Applications, (Springer-Verlag, New York, 1980).

[54] E. T. Whittaker and G. N. Watson, A course of modern analysis, (Cambridge Univ. Press, Cambridge, 1980).

[55] F. Correa, V. Jakubsky and M. S. Plyushchay, "Aharonov-Bohm effect on $\mathrm{AdS}_{2}$ and nonlinear supersymmetry of reflectionless Poschl-Teller system," Annals Phys. 324, 1078 (2009) arXiv:0809.2854 [hep-th].

[56] D. J. Fernandez, B. Mielnik, O. Rosas-Ortiz and B. F. Samsonov, "The Phenomenon of Darboux Displacements," Phys. Lett. A 294, 168 (2002) arXiv:quant-ph/0302204. 
[57] S. S. Shei, "Semiclassical Bound States In A Model With Chiral Symmetry," Phys. Rev. D 14, 535 (1976).

[58] G. V. Dunne and M. Shifman, "Duality and Self-Duality (Energy Reflection Symmetry) of Quasi-Exactly Solvable Periodic Potentials," Annals Phys. 299, 143 (2002). arXiv:hep-th/0204224.

[59] M. Faux and D. Spector, "A BPS Interpretation of Shape Invariance," J. Phys. A 37, 10397 (2004). arXiv:quant-ph/0401163.

[60] V. Z. Enolskii, 'On solutions in elliptic functions of integrable nonlinear equations", Phys. Lett. A 96, 327 (1983); V. M. Buchstaber, V. Z. Enolskii and D. V. Leikin, "Hyperelliptic Kleinian functions and applications", in Solitons, geometry, and topology: on the crossroad, Amer. Math. Soc. Transl. Ser. 2, 179, 1 (1997), arXiv:solv-int/9603005; J. C.Eilbeck, V. Z. Enolskii and E. Previato, "Spectral Curves of Operators with Elliptic Coefficients", SIGMA 3, 045 (2007), math-ph/0703038

[61] M. V. Babich, A. I. Bobenko and V. B. Matveev, "Solutions of nonlinear equations integrable in Jacobi theta functions by the method of the inverse problem, and symmetries of algebraic curves", Math. USSR Izv. 26, 479 (1986).

[62] C. Boehmer, M. Thies and K. Urlichs, "Tricritical behavior of the massive chiral Gross-Neveu model," Phys. Rev. D 75, 105017 (2007) arXiv:hep-th/0702201; C. Boehmer, F. Karbstein and M. Thies, "Baryons in the large N limit of the massive two-dimensional Nambu-Jona-Lasinio model," Phys. Rev. D 77, 125031 (2008) arXiv:0803.1369 [hep-th].

[63] B. Bringoltz, "Chiral crystals in strong-coupling lattice QCD at nonzero chemical potential," JHEP 0703, 016 (2007) arXiv:hep-lat/0612010, "Solving two-dimensional large-N QCD with a nonzero density of baryons and arbitrary quark mass," arXiv:0708.0589 [hep-lat].

[64] D. Nickel and M. Buballa, "Solitonic ground states in (color-) superconductivity," Phys. Rev. D 79, 054009 (2009) arXiv:0811.2400 [hep-ph]; D. Nickel, "How many phases meet at the chiral critical point?" arXiv:0902.1778 [hep-ph].

[65] E. Antonyan, J. A. Harvey, S. Jensen and D. Kutasov, "NJL and QCD from string theory," arXiv:hep-th/0604017; E. Antonyan, J. A. Harvey and D. Kutasov, "The Gross-Neveu model from string theory," Nucl. Phys. B 776, 93 (2007) arXiv:hep-th/0608149.

[66] A. Parnachev and D. A. Sahakyan, "Chiral phase transition from string theory," Phys. Rev. Lett. 97, 111601 (2006) arXiv:hep-th/0604173.

[67] A. Basu and A. Maharana, "Generalized Gross-Neveu models and chiral symmetry breaking from string theory," Phys. Rev. D 75, 065005 (2007) arXiv:hep-th/0610087.

[68] J. L. Davis, M. Gutperle, P. Kraus and I. Sachs, "Stringy NJL and Gross-Neveu models at finite density and temperature," JHEP 0710, 049 (2007) arXiv:0708.0589 [hep-th].

[69] For details and proof see Ref. 31]

[70] For its applications see [3, 4, 46] and references therein.

[71] Hidden nonlinear supersymmetry of the reflectionless Pöschl-Teller system can be explained in terms of the Aharonov-Bohm effect for non-relativisitc particle on $\operatorname{AdS}(2)$, see [55].

[72] We note that the spectrum also reveals a symmetry $E\left(2 \mathbf{K}^{\prime}-\theta\right) \rightarrow-E\left(2 \mathbf{K}^{\prime}+\theta\right)$ [and $\mathcal{E}(\pi-\theta) \rightarrow-\mathcal{E}(\pi+\theta)$ in the nonperiodic case]. This symmetry is not independent, but is a consequence of the duality relation and the invariance of the spectrum under reflection with respect to $\theta=4 \mathbf{K}^{\prime}[\theta=2 \pi]$. The latter, in turn, follows from invariance of the Hamiltonian [4.38) [4.7] ] under the change $\theta \rightarrow-\theta$ and its quasi-invariance [invariance] under the shift for the period $8 \mathbf{K}^{\prime}[4 \pi]$. 